COMMUNICATIONS IN

ANALYSIS AND GEOMETRY

Volume 4, Number 4, 547-587, 1996

\title{
Denjoy-Ahlfors Theorem for Harmonic Functions on Riemannian Manifolds and External Structure of Minimal Surfaces
}

\author{
Vladimir Miklyukov and Vladimir TKaCheV
}

\begin{abstract}
We extend the well-known Denjoy-Ahlfors theorem about the number of different asymptotic tracts of a holomorphic function to subharmonic functions on arbitrary Riemannian manifolds. We obtain some versions of the Liouville theorem for $\alpha$-harmonic functions without the geodesic completeness requirement on a manifold. Moreover, the upper estimate of the topological index of the height function of a minimal surface in $\mathbf{R}^{n}$ has been established and, as a consequence, a new prove of the Bernstein's theorem has been derived. Other applications to the theory of minimal surfaces are also discussed.
\end{abstract}

\section{Introduction.}

The present study arose from an attempt to develop efficient methods for investigation of external properties of higherdimensional minimal surfaces. The main difficulty of this theory is a lack of any representation for such surfaces to be analogous of the well known Weierstrass one. On the other hand, the coordinate functions of minimal immersions are harmonic ones in the inner metric, and from this point of view the minimal submanifolds can regarded as the special type Riemannian manifolds $M$ having a system of harmonic functions $\left\{f_{1}(m), \ldots, f_{n}(m)\right\}$ with some structure conditions like the following one

$$
\sum_{k=1}^{n}\left|\nabla f_{k}(m)\right|^{2}=\operatorname{dim} M .
$$

\footnotetext{
${ }^{1}$ This paper was supported by RFRF, project 93-011-176 and by SanktPeterburg University grant, project 95-0-1.9-34
} 
Moreover, the minimality condition is a source of the numerous subharmonic algebraic combinations of the coordinate functions and the Gauss map on $M$.

This property along with suitable functional assertions concerning subharmonic functions allows us to derive an information about the geometrical structure of minimal surfaces. We mention the substantial papers of Yau [37], Cheng and Yau [4]-[5] realizing this way on the complete higherdimensional Riemannian manifolds with restrictions on the Ricci curvature. The main tool was the maximum principle for the elliptic type PDE (see also [11], [12]). On the other hand, the geometrical applications of the Liouville and Phragmen-Lindelöff type theorems have been obtained in [20]-[22], [24],[25] by using of methods the quasiconformal maps theory. We notice that such theorems can regarded as consequences of an appropriate form of the general Denjoy-Ahlfors theorem on Riemannian manifolds.

The classical Denjoy-Ahlfors theorem states that the order of growth of an entire holomorphic function $f(z)$ gives the upper bound on the number $\rho$ of different asymptotic values of $f(z)$. More precisely, let $w=f(z)$ be such a function. A family of domains $\{\mathcal{D}(\tau)\}$ is called the asymptotic tract of $w=f(z)$ if

a) every $\mathcal{D}(\tau)$ is an open component of the set

$$
\{z \in \mathbf{C}:|f(z)|>\tau>0\}
$$

b) for all $\tau_{2}>\tau_{1}>0$ holds $\mathcal{D}\left(\tau_{1}\right) \supset \mathcal{D}\left(\tau_{2}\right)$ and $\cap_{\tau} \overline{\mathcal{D}}(\tau)=\emptyset$.

Two asymptotic tracts $\mathcal{D}^{\prime}(\tau)$ and $\mathcal{D}^{\prime \prime}(\tau)$ are different if for a sufficient large $\tau>0$ we have $\mathcal{D}^{\prime}(\tau) \cap \mathcal{D}^{\prime \prime}(\tau)=\emptyset$.

Then the mentioned inequality is

$$
\limsup _{r \rightarrow+\infty} \frac{\log \log M(r)}{\log r} \geq \frac{1}{2} \rho
$$

where $M(r)$ is the maximum modulus of $f(z)$ on $|z|=r$

It is well known [13] that this assertion gives an estimate of the number of different asymptotic tracts of the holomorphic function by its lower order. This terminology goes back to [19] and seems to us more preferable by virtue of its adoptation for geometrical applications.

In this paper we extend the Denjoy-Ahlfors theorem to Riemannian manifolds. In addition, the geometrical approach enables us to apply the technique developed to the theory of minimal surfaces. We give now a sketch of our method. Let $M$ be a noncompact $p$-dimensional Riemannian manifold and $(M, u)$ be a $C^{2}$-surface given by an immersion $u(m): M \rightarrow \mathbf{R}^{n}$. 
The surface $\mathcal{M} \equiv(M, u)$ is called minimal if the mean curvature vector is identically zero. If $\mathcal{M}$ is the minimal surface then for an arbitrary vector $e \in \mathbf{R}^{n}$ the corresponding coordinate function $f(m)=\langle u, e\rangle(m)$ is harmonic with respect to the inner metric of $\mathcal{M}([17])$. Then the asymptotic tracts of $f(m)$ can be identified with the noncompact components which one can cut off from $u(M)$ by the hyperplanes $\langle u, e\rangle=$ const. On the other hand, there exists an a priori upper bound on the order of $f(m)$ in terms of the growth of the distance function in $\mathbf{R}^{n}$. Hence, the Denjoy-Ahlfors theorem applied to $f(m)$ on a minimal surface $\mathcal{M}$ yields some relation between the inner geometric characteristics of $\mathcal{M}$ and the number of pieces which one can cut off from this surface by the hyperplanes.

The first three sections of the paper are concerned with the generalizations of the classical Denjoy-Ahlfors theorem and its consequences on arbitrary not necessary geodesically complete manifolds. In Section 3 we derive the first order differential inequality on the Dirichlet integral of a subharmonic function in terms of the $N$-means of fundamental frequency of its level sets. This inequality leads to two different types of asymptotic tracts of harmonic functions on topologically nontrivial manifolds. Namely, there are harmonic functions with compact level sets generating the singular asymptotic tracts. The corresponding theory is rather complicated by virtue of the additional term in the main differential inequality. This case is closely linked with the tubular type minimal surfaces. In Section 4 we concern the singular tracts.

It was recently shown in [35] that there exists a finite or infinite limit

$$
V_{p}(\mathcal{M})=\frac{1}{\omega_{p}} \lim _{R \rightarrow \infty} \frac{1}{\ln R} \int_{M_{a}(R)} \frac{d x}{|x-a|^{p}},
$$

where $M_{a}(R)$ is the part of the minimal surface $\mathcal{M}$ situated inside of the punctured ball $\{1<|x-a|<R\}$. We call $V_{p}(\mathcal{M})$ the projective volume of $\mathcal{M}$. As follows from [35] this quantity does not depend on $a$ and the number of ends of $\mathcal{M}$ can be estimated by $c(p, n) V_{p}(\mathcal{M})$. Moreover, the numerous connections between $V_{p}(\mathcal{M})$ and the integral-geometrical means of $\mathcal{M}$ have been obtained there.

In the final section of the paper we use the developed methods to study the external structure of minimal surfaces with finite projective volume.

Theorem 0.1. Let $\mathcal{M}$ be a two-dimensional properly immersed minimal surface in $\mathbf{R}^{n}$ of finite topological type, $e$ is a regular direction and $x_{1}$ be the corresponding coordinate function. If $V_{2}(\mathcal{M})<+\infty$ then either $\mathcal{M}$ lies in 
the hyperplane $x_{1}=$ const, or the number of critical points $\left\{a_{i}\right\}$ of $x_{1}(m)$ is finite. Moreover, the following estimate of the topological index of $\mathcal{M}$ holds

$$
\sum_{j} \operatorname{ind}\left(a_{j}\right) \leq V_{2}(\mathcal{M})-\chi(M),
$$

where $\chi(M)$ is the Eulerian characteristic of $M$.

When $\mathcal{M}$ is homeomorphic to a sphere with a finite number of points removed Theorem 1 was announced without proof in [23].

Let $\mathcal{M}$ be a two-dimensional simply connected surface. By $n\left(t e^{i \theta}\right)$ we denote the multiplicity of the orthogonal projection of $\mathcal{M}$ onto a fixed 2plane $V$ at a point $z=t e^{i \theta}$. Then the following result extends the well-known Bernstein's theorem

Theorem 0.2. Let $\mathcal{M}$ be a two-dimensional minimal properly immersed plane in $\mathbf{R}^{n} 3$ and $\mathcal{M}$ be proper with respect to $V$. Then either $\mathcal{M}$ is some plane in $\mathbf{R}^{n} 3$, or

$$
\liminf _{R \rightarrow \infty} \frac{1}{\ln R} \int_{1}^{R} \frac{d t}{t} \int_{0}^{2 \pi} n\left(t e^{i \theta}\right) d \theta \geq 8 .
$$

We establish the most of preliminary assertions in the form to be suitable for $\alpha$-subharmonic functions. In particularly, this allows us to apply these results to $\alpha$-minimal surfaces (see the precise definition in Section $\mathrm{E}$ and also [36])

Finally, we agree upon the notations. We assume henceforth that $M$ is a $p$-dimensional noncompact orientiable Riemannian $C^{2}$-manifold. By $\partial \mathcal{O}$ we denote the boundary of a (sub)manifold $\mathcal{O}$. If $\partial M$ is a nonempty set we assume that it is piecewise smooth. By $T_{m}(M),\langle u, e\rangle X Y$ and $\nabla$ we denote the tangent space to $M$ at $m \in M$, the scalar product of two vectors $X, Y \in T_{m}(M)$ and Levi-Civita connection on $M$ respectively.

Let $\mathcal{M}=(M, u)$ be a surface defined by a $C^{2}$-immersion $u(m): M \rightarrow$ $\mathbf{R}^{n}, p<n$. Then we consider the Riemannian connection $\nabla$ to be induced by the immersion $u(m)$. Let $\bar{\nabla}$ be the the Euclidean connection in $\mathbf{R}^{n}$ and $N_{m}(M)$ be the normal space at $m$. Then, given $\xi \in N_{m}(M), X, Y \in T_{m}(M)$ we have the Gauss formulas

$$
\left(\bar{\nabla}_{X} Y\right)^{\top}=\nabla_{X} Y, \quad\left(\bar{\nabla}_{X} Y\right)^{\perp}=B(X, Y),
$$


and the Weingarten formulas

$$
\left(\bar{\nabla}_{X} \xi\right)^{\top}=-A^{\xi}(X), \quad\left(\bar{\nabla}_{X} \xi\right)^{\perp}=\nabla_{X} \xi .
$$

Here the top symbols $T$ and $\perp$ denote the orthogonal projections on $T_{m}(M)$ and $N_{m}(M)$ respectively. Here, by $B$ and $A$ we denote the second fundamental form and Weingarten map respectively. Then the following relation holds

$$
\langle u, e\rangle A^{\xi}(X) Y=\langle u, e\rangle B(X, Y) \xi .
$$

We are also droping everywhere the Lebesgues measure designation in the integrals we use.

Aknowledgements: The authors thank the referees for many helpful suggestions that greatly improved the presentation of this paper and Ms.Daniela Santoro for her permanent assistance.

\section{Subharmonic and exhausting functions.}

1.1. We remind that the divergence of a smooth vector field $X$ is defined to be

$$
\operatorname{div} X=\sum_{i=1}^{p}\langle u, e\rangle \nabla_{E_{i}} X E_{i}
$$

where the last sum does not depend on choice of the orthonormal basis $\left\{E_{i}\right\}$ of $T_{m} M$.

Let $\alpha>1$ be a fixed real parameter. We define the differential operator

$$
\Delta_{\alpha} f=\operatorname{div}\left(|\nabla f|^{\alpha-2} \nabla f\right)=|\nabla f|^{\alpha-3}(|\nabla f| \Delta f+(\alpha-2)\langle u, e\rangle \nabla f \nabla|\nabla f|)
$$

where $\Delta \equiv \Delta_{2}$ is the ordinary Laplace operator on $M$.

We denote by $\mathcal{Z}(f)$ the set of all critical points of a function $f(m)$.

Definition 1. A smooth function $f(m)$ is said to be $\alpha$-subharmonic if the inequality

$$
\Delta_{\alpha} f(m) \geq 0
$$

holds everywhere in $M \backslash \mathcal{Z}(f)$. If the boundary $\partial M$ is nonempty we assume that everywhere on $\partial M$ holds

$$
\langle u, e\rangle \nabla f(m) \nu(m)=0
$$

where $\nu(m)$ is the outward normal to the boundary $\partial M$. 
Definition 2. If (1.1) turned into equality the function $f(m)$ is called $\alpha$-harmonic.

1.2. Let $h(m): M \rightarrow\left(0 ; h_{0}\right)$ be a continuous function of $C^{\infty}\left(M \backslash \Sigma_{0}\right)$, where $\Sigma_{0}$ is the null-level set of $h(m)$. We introduce for $t \in\left(0 ; h_{0}\right)$ an $h$-ball as

$$
B_{h}(t)=\{m \in M: h(m)<t\},
$$

and an $h$-sphere as

$$
\Sigma_{h}(t)=\{m \in M: h(m)=t\} .
$$

Definition 3. The function $h(m)$ is called an exhausting function on $M$ if

1. $B_{h}(t)$ is precompact for all $t \in\left(0 ; h_{0}\right)$;

2. $\lim _{k \rightarrow \infty} h\left(m_{k}\right)=h_{0}$ along the every sequence $m_{k} \in M$ without accumulation points in $M \cup \partial M$;

3. $|\nabla h(m)|>0$ almost everywhere in $M$.

Example 1. Let $M$ be a complete Riemannian manifold. Then the distance function $h(m)=\operatorname{dist}\left(m_{0}, m\right)$ is an exhausting function on $M$. Furthermore, at every regular point of $h(m)$ we have $|\nabla h(m)|=1$.

The following assertion describes the store of exhausting functions on minimal submanifolds in $\mathbf{R}^{n}$.

Lemma 1.1. Let $\mathcal{M}=(M, u)$ be a minimal surface in $\mathbf{R}^{n}$ and $\varphi(x)$ be a smooth function defined on $\mathbf{R}^{n}$. We assume that the restriction $h(m)=$ $\varphi \circ u(m)$ satisfies (1) and (2) in Definition 3 and there exists a smooth positive function $\psi(t)$ for which the composition $\psi \circ \varphi$ is a strong convex function. Then $h(m)$ is an exhausting function on $M$.

Proof. We needed only to prove that $|\nabla h(m)|>0$ almost everywhere in $M$. If this fail then there exists a closed set $\mathcal{Z}(h)$ of positive Lebesgues measure such that $|\nabla h(m)|=0$ if $m \in \mathcal{Z}(h)$. This would implied existence of a point $m_{0} \in \mathcal{Z}(h)$ such that the contingency of $\mathcal{Z}(h)$ at $m_{0}$ coincides with $T_{m_{0}} M$. Let $\left\{E_{k}(m): k=1, \ldots, p\right\}$ be any orthonormal system which is well defined in some neighbourhood of $m_{0}$. In view of degeneracy of the 
gradient tangent component $\nabla h(m)=(\bar{\nabla} 1 \varphi \circ u)^{\top}=0$ everywhere on $\mathcal{Z}(h)$, we have for its normal component

$$
(\bar{\nabla} 1 \varphi \circ u)^{\perp}=\bar{\nabla} 1(\varphi \circ u), \quad m \in \mathcal{Z}(h) .
$$

By the definition of the Weingarten map we notice that

$$
\begin{gathered}
A^{\zeta}\left(E_{j}\right)=-\left(\bar{\nabla} 1_{E_{j}} \bar{\nabla} 1(\psi \circ \varphi)\right)^{\top}=-\left[\sum_{k=1}^{n} \bar{\nabla} 1_{E_{j}}\left(e_{k} \frac{\partial(\psi \circ \varphi)}{\partial x_{k}}\right)\right]^{\top} \\
=-\sum_{k=1}^{n}\langle u, e\rangle E_{j} e_{i}^{\top} e_{k}^{\top} \frac{\partial^{2}(\psi \circ \varphi)}{\partial x_{k} \partial x_{j}}=
\end{gathered}
$$

where $\zeta=[\bar{\nabla} 1(\psi \circ \varphi)]^{\perp}$ and $\left\{x_{k}\right\}$ are the coordinate functions in $\mathbf{R}^{n}$ which are dual to the basis $\left\{e_{k}\right\}$. Taking the trace in the last formula yields at $m_{0}$

$$
\begin{aligned}
\langle u, e\rangle H \bar{\nabla} 1(\psi \circ \varphi) & =-\sum_{i, k=1}^{n} \sum_{j=1}^{n}\langle u, e\rangle E_{j} e_{i}^{\top}\langle u, e\rangle E_{j} e_{k}^{\top} \frac{\partial^{2}(\psi \circ \varphi)}{\partial x_{k} \partial x_{j}}= \\
& =-\sum_{i, k=1}^{n}\langle u, e\rangle e_{k}^{\top} e_{i}^{\top} \frac{\partial^{2}(\psi \circ \varphi)}{\partial x_{k} \partial x_{i}} .
\end{aligned}
$$

On the other hand, the Hessian

$$
\left\|\frac{\partial^{2}(\psi \circ \varphi)}{\partial x_{k} \partial x_{i}}\right\|
$$

is a positively definite quadratic form at $m_{0}$ by virtue of strong convexity of $\psi \circ \varphi$. Taking into consideration vanishing of $H \equiv 0$ and the last identity we arrive at

$$
\langle u, e\rangle e_{k}^{\top} e_{i}^{\top}=0,
$$

for all $i, k \leq n$. Substituting $i=k$ gives $\left|e_{k}^{\top}\right|=0$ and summing

$$
0=\sum_{k=1}^{n}\left|e_{k}^{\top}\right|^{2}=\operatorname{dim} M=p,
$$

we obtain the contradiction and the lemma is proved.

Example 2. Let $(M ; u)$ be a properly immersed surface in $\mathbf{R}^{n}$. Then $\varphi(m)=|u(m)|$ is an exhausting function on $M$. 
From now on we shall assume that an exhausting function $h$ satisfies the boundary condition (1.2) if the boundary $\partial M$ is not empty.

1.3. Let $f(m)$ be a different from a constant function which satisfies the maximum principle, i.e. for every open set $U \subset M$ with compact closure

$$
\max _{m \in \partial U} f(m)=\max _{m \in \bar{U}} f(m) .
$$

We notice that (1.3) valid for arbitrary $\alpha$-subharmonic function. Really, it follows from the general maximum principle for the elliptic type PDE's (see [8]).

Definition 4. A family of domains $\{D(\tau): \tau \in(\alpha, \beta)\}$ on $M$ is said to be the asymptotic tract of $f(m)$ if:

(i) for every $\tau \in(\alpha, \beta)$ the domain $D(\tau)$ is not empty open component of the superlevel set $\{m \in M: f(m)>\tau\}$;

(ii) $D\left(\tau_{1}\right) \supset D\left(\tau_{2}\right)$ for all $\tau_{1}<\tau_{2}$ from the interval $(\alpha, \beta)$;

(iii) either $\beta=+\infty$, or for some $\tau \in(\alpha, \beta)$ the set

$$
D(\tau) \cap\{m \in M: f(m)>\beta\}
$$

is empty.

It follows from (1.3), (i) and (iii) that every domain $D(\tau)$ has noncompact closure.

We say that two asymptotic tracts are different if there exist such $\tau_{1} \in$ $\left(\alpha_{1}, \beta_{1}\right)$ and $\tau_{2} \in\left(\alpha_{2}, \beta_{2}\right)$ that $D\left(\tau_{1}\right) \cap D\left(\tau_{2}\right)=\emptyset$.

Let $h(m)$ be an exhausting function on $M$ and $\{D(\tau): \tau \in(\alpha, \beta)\}$ be an asymptotic tract of $f(m)$.

Definition 5. The tract $\{D(\tau)\}$ is called regular if there exist $\tau_{0} \in(\alpha, \beta)$ and a system of nonintersecting intervals $\triangle_{1} k \subset\left(0, h_{0}\right)$ tending to $h_{0}$, i.e. $h_{0} \in \cup_{k} \overline{\triangle_{1} k}$ so that the set

$$
D\left(\tau_{0}\right) \cap \Sigma_{h}(t)
$$

does not consist of any cycles for all values $t \in \cup_{k} \Delta_{k}$. Otherwise, we shall say that $\{D(\tau)\}$ is the singular asymptotic tracts.

\section{The fundamental frequency, its $N$-means and the Dirichlet integral.}

2.1. Let $\Sigma$ be a compact $p$-dimensional manifold. Further we define the weighted fundamental frequency for the subsets of $\Sigma$ which consist of a finite 
system of nonoverlapping $(p-1)$-dimensional compact submanifolds $\mathcal{O}_{j}$ of $\Sigma$ with or without a boundary. Such a set we call to be simple set (of $\Sigma$ ). We notice, that the $h$-sphere $\Sigma_{h}(t)$ is a simple set for every regular value $t$ of $h(m)$.

Let $U$ be a simple set consisting of components $\mathcal{O}_{1}, \mathcal{O}_{2}, \ldots, \mathcal{O}_{k}$ and $\theta$ be a positive in essential smooth function on $U$. We say that a Lipschitz function $\varphi$ is admissible for $U$, or $\varphi \wedge U$, if for each component $\mathcal{O}_{j}$ with nonempty boundary $\partial \mathcal{O}_{j}:\left.\varphi\right|_{\partial \mathcal{O}_{j}}=0$ and the equality

$$
\int_{\mathcal{O}_{j}}|\varphi(m)|^{\alpha-2} \varphi(m) \theta(m)=0
$$

holds if $\partial \mathcal{O}_{j}=\emptyset$. Further we say open and cyclic components for the components $\mathcal{O}_{j}$ with or without a boundary respectively.

In Section 3 we use the following helpful property. Let $\mathcal{O}_{j}$ is a cyclic component and $\varphi(m)$ be an arbitrary Lipschitz function on $\mathcal{O}_{j}$. Then the improvement function $\varphi(m)-\xi$ will be already an admissible function for $\mathcal{O}_{j}$ if $\xi \equiv \phi_{\alpha}\left(\varphi, \mathcal{O}_{j}\right)$ is the unique root of the following equation

$$
\int_{\mathcal{O}_{j}}|\nabla f| \xi-\varphi(m)^{\alpha-2}(\xi-\varphi(m)) \theta(m)=0,
$$

where the integration considered over the $(p-1)$-dimensional Hausdorff measure on $\mathcal{O}_{j}$. One can show that the last integral is increasing function on $\xi$, and hence the quantity $\phi_{\alpha}\left(\varphi, \mathcal{O}_{j}\right)$ is well defined. The explicit expression for $\phi_{\alpha}\left(\varphi, \mathcal{O}_{j}\right)$ is known only for $\alpha=2$ :

$$
\phi_{2}\left(\varphi, \mathcal{O}_{j}\right)=\frac{\int_{\mathcal{O}_{j}} \varphi(m) \theta(m)}{\int_{\mathcal{O}_{j}} \theta(m)} .
$$

Definition 6. The quantity

$$
\lambda_{\alpha, \theta}(U)=\inf _{\varphi \wedge U}\left[\frac{\int_{U}|\nabla f| \nabla \varphi^{\alpha} \theta^{-1}}{\int_{U}|\nabla f| \varphi^{\alpha} \theta^{\alpha-1}}\right]^{\frac{1}{\alpha}}
$$

is called a fundamental $\alpha$-frequency of a simple set $U$ with respect to the weight $\theta$. We also use the reduced fundamental frequency defined by

$$
\lambda_{\alpha, \theta}^{\star}(U)=\lambda_{\alpha, \theta}(U)
$$

if $U$ hasn't any cyclic component and $\lambda_{\alpha, \theta}^{\star}(U)=0$ otherwise. 
Example 3. If $\theta \equiv 1$ on $U, \alpha=2$ and $U$ is a domain with the piecewise smooth nonempty boundary, then $\lambda_{2, \theta}^{2}(U)$ is the first nontrivial eigenvalue of the classical variational Dirichlet problem. Other words,

$$
\Delta_{\Sigma} f+\lambda_{2, \theta}^{2}(U) f=0
$$

everywhere in $U$ for some positive function $f(m)$ equal to zero on $\partial U$. Here $\Delta_{\Sigma}$ is the Laplace operator on $\Sigma$.

Example 4. Let now $\Sigma=\Sigma(\tau)$ be the submanifold in $M$ given by the equation $h(m)=\tau$. Then it turns out that the most natural choice of weight function $\theta(m)$ is $|\nabla f| \nabla h(m)$. We write $\lambda_{\alpha, 1}(2) h \mathcal{O}$ instead of $\lambda_{\alpha, 1}(2)|\nabla f| \nabla h \mathcal{O}$.

Now we formulate some basic properties of the fundamental $\alpha$-frequency $\lambda^{(\star)}(U) \equiv \lambda_{\alpha, \theta}^{(\star)}(U)$.

Lemma 2.1. If $U_{1} \subset U_{2}$, then $\lambda^{\star}\left(U_{1}\right) \geq \lambda^{\star}\left(U_{2}\right)$, i.e. $\lambda^{\star}$ is nonincreasing function of a set. If $U$ consists of open components $\mathcal{O}_{1}, \ldots, \mathcal{O}_{k}$ only then

$$
\lambda(U)=\min _{1 \leq i \leq k} \lambda\left(\mathcal{O}_{i}\right)
$$

Proof. If $U_{1}$ contains a cyclic component then $U_{2}$ does too. It follows that $\lambda^{\star}\left(U_{1}\right)=\lambda^{\star}\left(U_{2}\right)=0$ in this case. On the other hand, if $U_{2}$ is without cyclics then such does $U_{1}$ too. Hence monotonicity of $\lambda^{\star}$ follows from the fact that $\varphi \wedge U_{1}$ implies $\varphi \wedge U_{2}$.

To prove the second property we fix arbitrary functions $\varphi_{i} \wedge \mathcal{O}_{i}$ and set $\varphi(m)=\varphi_{i}(m)$ for $m \in \mathcal{O}_{i}$. Hence

$$
\lambda^{\alpha}\left(\mathcal{O}_{i}\right) \int_{\mathcal{O}_{i}}|\nabla f| \varphi^{\alpha} \leq \int_{\mathcal{O}_{i}}|\nabla f| \nabla \varphi^{\alpha}
$$

Summing of all this inequalities

$$
\min _{1 \leq i \leq k} \lambda^{\alpha}\left(\mathcal{O}_{i}\right) \sum_{i=1}^{k} \int_{\mathcal{O}_{i}}|\varphi|^{\alpha} \leq \sum_{i=1}^{k} \int_{\mathcal{O}_{i}}|\nabla f| \nabla \varphi^{\alpha}
$$

we obtain $\min _{1 \leq i \leq k} \lambda\left(\mathcal{O}_{i}\right) \leq \lambda(\mathcal{O})$. The reverse inequality follows now from the first assertion of the lemma.

The following assertion is an extension of the known for case $\alpha=2$ lower estimation [4] of the fundamental frequency of an open set. Our proof is different from [4] where the main tool is the maximum principle for solutions of the elliptic type PDE's. 
Lemma 2.2. Let $\alpha>1$ and $f(m)>0$ be a function of $C^{2}(U)$ such that $\mathcal{Z}(f)=\emptyset$. Then

$$
\left(\lambda_{\alpha, 1}(2) \theta U\right)^{\alpha} \geq \inf _{U}\left[-\frac{1}{(f \theta)^{\alpha-1}} \operatorname{div} \frac{|\nabla f| \nabla f^{\alpha-2} \nabla f}{\theta}\right] .
$$

Here $\nabla$, div are considered with respect to the inner metric of $U$.

Proof. We fix a function $\varphi(m) \wedge U$ and denote by $\beta$ the right side of (2.3). Then from positivity of $f(m)$ we have everywhere in $U$

$$
\operatorname{div}\left(|\nabla f| \nabla f^{\alpha-2} \frac{\nabla f}{\theta}\right)+\beta(f \theta)^{\alpha-1} \leq 0
$$

and hence,

$$
\begin{gathered}
\theta^{\alpha-1} \varphi^{\alpha} \beta=\frac{\varphi^{\alpha} \beta}{f^{\alpha-1}}(f \theta)^{\alpha-1} \leq-\frac{\varphi^{\alpha}}{f^{\alpha-1}} \operatorname{div}\left(|\nabla f| \nabla f^{\alpha-2} \frac{\nabla f}{\theta}\right)= \\
-\operatorname{div}\left(\frac{|\nabla f| \nabla f^{\alpha-2} \varphi^{\alpha}}{\theta f^{\alpha-1}} \nabla f\right)+\frac{|\nabla f| \nabla 1 f^{\alpha-2}}{\theta f^{\alpha}}\langle u, e\rangle \nabla 1 f \alpha f \varphi^{\alpha-1} \nabla 1 \varphi-(\alpha-1) \varphi^{\alpha} \nabla 1 f \leq
\end{gathered}
$$

$-\operatorname{div}\left(\frac{|\nabla f| \nabla f^{\alpha-2} \varphi^{\alpha}}{\theta f^{\alpha-1}} \nabla f\right)+\alpha \frac{(\varphi|\nabla f| \nabla f)^{\alpha-1}}{\theta f^{\alpha-1}}|\nabla f| \nabla \varphi-(\alpha-1) \frac{|\nabla f| \nabla 1 f^{\alpha} \varphi^{\alpha}}{\theta f^{\alpha}}$.

Let $U$ be decomposed into collection of the components $U_{1}, \ldots, U_{k}$ with nonempty boundaries and the closed components $\mathcal{O}_{1}, \ldots, \mathcal{O}_{s}$. By virtue of the $\varphi(m) \wedge U$ we have $\left.\varphi\right|_{\partial U_{i}}=0$. Using the Stokes' formula and (2.4) we arrive at the inequality

$$
\beta \int_{U} \theta^{\alpha-1} \varphi^{\alpha} \leq \alpha \int_{U} \frac{(\varphi|\nabla f| \nabla 1 f)^{\alpha-1}}{\theta f^{\alpha-1}}|\nabla f| \nabla 1 \varphi-(\alpha-1) \int_{U} \frac{|\nabla f| \nabla 1 f^{\alpha} \varphi^{\alpha}}{\theta f^{\alpha}} .
$$

Applying the Cauchy's integral inequality we obtain

$$
\begin{gathered}
\beta \int_{U} \theta^{\alpha-1} \varphi^{\alpha} \leq \alpha\left[\int_{U} \frac{|\nabla f| \nabla 1 f^{\alpha} \varphi^{\alpha}}{\theta f^{\alpha}}\right]^{\frac{\alpha-1}{\alpha}}\left[\int_{U}|\nabla f| \nabla 1 \varphi^{\alpha} \frac{1}{\theta}\right]^{\frac{1}{\alpha}}- \\
(\alpha-1) \int_{U} \frac{|\nabla f| \nabla 1 f^{\alpha} \varphi^{\alpha}}{\theta f^{\alpha}}
\end{gathered}
$$


Given $k>1$, we can find $\varphi(m) \wedge U$ such that

$$
\int_{U} \frac{|\nabla f| \nabla 1 \varphi^{\alpha}}{\theta} \leq k \lambda^{\alpha}(U) \int_{U} \varphi^{\alpha} \theta^{\alpha-1} .
$$

where we abbreviate $\lambda(U)=\lambda_{\alpha, 1}(2) \theta U$. It follows from (2.5)

$$
\begin{gathered}
\beta \int_{U} \varphi^{\alpha} \theta^{\alpha-1} \leq \alpha \lambda(U) k^{\frac{1}{\alpha}}\left[\int_{U} \frac{|\nabla f| \nabla 1 f^{\alpha} \varphi^{\alpha}}{\theta f^{\alpha}}\right]^{\frac{\alpha-1}{\alpha}}\left[\int_{U} \varphi^{\alpha} \theta^{\alpha-1}\right]^{\frac{1}{\alpha}}- \\
(\alpha-1) \int_{U} \frac{|\nabla f| \nabla 1 f^{\alpha} \varphi^{\alpha}}{\theta f^{\alpha}} .
\end{gathered}
$$

Hence we obtain

$$
A(\xi) \equiv \beta \xi^{\alpha}-\alpha k^{\frac{1}{\alpha}} \lambda(U) \xi+(\alpha-1) \leq 0,
$$

where

$$
\xi=\left[\int_{U} \frac{|\nabla f| \nabla 1 f^{\alpha} \varphi^{\alpha}}{\theta f^{\alpha}}\right]^{-\frac{1}{\alpha}}\left[\int_{U} \varphi^{\alpha} \theta^{\alpha-1}\right]^{\frac{1}{\alpha}}
$$

Now finding by standard arguments the minimum of $A(\xi)$ over all $\xi \geq 0$ we conclude that

$$
A(\xi) \geq(\alpha-1)\left[\frac{\beta}{\lambda(U) k^{\frac{1}{\alpha}}}\right]^{\frac{1}{\alpha-1}}-(\alpha-1) k^{\frac{1}{\alpha}} \lambda(U)
$$

and by virtue of (2.6) we obtain

$$
\beta \leq \lambda^{\alpha}(U) k
$$

Taking into account the condition on $k$ we complete the proof of Lemma B2.

2.2. We consider a $(p-1)$-dimensional subset $\mathcal{O} \subset \Sigma$ and an integer $N \geq 1$. Let us introduce

$$
\lambda(\mathcal{O}, N) \equiv \lambda_{\alpha, \theta}(\mathcal{O} ; N)=\inf \frac{1}{N} \sum_{i=1}^{N} \lambda_{\alpha, \theta}^{\star}\left(\mathcal{O}_{i}\right),
$$

where the infimum is taken over all systems $\left\{\mathcal{O}_{i}\right\}_{i=1}^{N}$ consisting of $N$ pairwise nonoverlapping simple subsets $\mathcal{O}_{i} \subset \mathcal{O}$. 
Definition 7. The quantity $\lambda(\mathcal{O}, N)$ is called $N$-mean of the fundamental frequency of $\mathcal{O}$.

Remark 1. It is clear from the definition that $\lambda(\mathcal{O} ; N)=0$ if $\mathcal{O}$ contains $N$ or more cyclic components. Moreover, we give without proof the following elementary property of this characteristic (see [21]),

$$
\lambda_{\alpha, 1}(2) \theta \mathcal{O} ; 1 \leq \ldots \leq \lambda_{\alpha, 1}(2) \theta \mathcal{O} ; N \leq \ldots
$$

Lemma 2.3. Let $\mathcal{O}$ be an open component, $\theta \equiv 1$ and $\varphi$ be an admissible for $\mathcal{O}$ function which satisfies equation $\Delta_{\alpha} \varphi=-\mu^{\alpha} \varphi|\nabla f| \varphi^{\alpha-2}$ in $\mathcal{O}$. Then

$$
\lambda_{\alpha, \theta}(\mathcal{O}, N) \leq \mu,
$$

where $N$ is the number components of the set $\mathcal{O}_{0}=\{m \in \mathcal{O}:|\nabla f| \varphi(m) \neq$ $0\}$.

Proof. The inequality follows immediately from the inequality $\lambda_{\alpha}\left(\mathcal{O}_{i}\right) \leq \mu$ which is true for each component $\mathcal{O}_{j} \subset \mathcal{O}_{0}$.

2.3. In this paragraph we give some estimates of the fundamental frequency of one-dimensional sets (families of curves) and their $N$-means. Let us consider a compact manifold $\Sigma, \operatorname{dim} \Sigma=1$. Then we have rather complete information about $N$-mean of the fundamental frequency.

Lemma 2.4. Given an open subset $\mathcal{O}=\cup_{i=1}^{s} \mathcal{O}_{i} \subset \Sigma$ such that $\partial \mathcal{O}_{i} \not \equiv$ $\emptyset, \mathcal{O}_{i} \cap \mathcal{O}_{j}=\emptyset$ and for any integer $N$ we have

$$
\lambda_{2, \theta}(\mathcal{O})=\pi\left[\max _{1 \leq i \leq s} \int_{\mathcal{O}_{i}} \theta(t) d t\right]^{-1} \geq \frac{\pi}{\int_{\mathcal{O}} \theta(t) d t}
$$

and

$$
\lambda_{2, \theta}(\mathcal{O} ; N) \geq \pi N\left[\max _{1 \leq i \leq s} \int_{\mathcal{O}} \theta(t) d t\right]^{-1}
$$

Proof. Without loss of generality we can assume that $\mathcal{O}$ is disjoint collection of intervals $\Delta_{1}, \Delta_{2}, \ldots, \Delta_{s}$ which are equipped by the natural parameterization with their lengths and let $|\nabla f| \Delta_{i}$ be the length of $\Delta_{i}$. We choose 
arbitrary $\varphi_{i}(t) \wedge \Delta_{i}$ and for $0 \leq t \leq|\nabla f| \Delta_{i}$ set

$$
\xi_{i}(t)=\int_{0}^{t} \theta_{i}(\tau) d \tau
$$

where $\theta_{i}(\tau)$ is the restriction of $\theta(t)$ on $\Delta_{i}$. Applying the Wirtinger's inequality we obtain

$$
\frac{\int_{0}^{|\nabla f| \Delta_{i}}|\nabla f| \varphi_{i}^{\prime}(t)^{2} \theta_{i}^{-1} d t}{\int_{0}^{|\nabla f| \Delta_{i}} \varphi_{i}(t)^{2} \theta_{i} d t}=\frac{\int_{0}^{\xi_{i}}|\nabla f| \psi_{i}^{\prime}(t)^{2} d t}{\int_{0}^{\xi_{i}} \psi_{i}(t)^{2} d t} \geq\left(\frac{\pi}{\xi_{i}}\right)^{2} .
$$

Here $\xi_{i}=\xi_{i}\left(|\nabla f| \Delta_{i}\right)$ and $\psi_{i}(\xi)=\varphi_{i} \circ t(\xi)$. The equality holds for $\psi_{i}(\xi)=\sin \left(\xi \pi / \xi_{i}\right)$ and hence by virtue of Lemma ??, (2.7) is proved.

In order to prove (2.8) we set $\epsilon>0$ and choose a decomposition $\mathcal{O}$ into the collection of the disjoint intervals $\delta_{i} \subset \mathcal{O}$ such that

$$
\epsilon+\lambda_{2, \theta}(\mathcal{O} ; N) \geq \frac{1}{N} \sum_{k=1}^{N} \lambda_{2, \theta}\left(\delta_{i}\right)=\frac{\pi}{N} \sum_{k=1}^{N}\left(\int_{\delta_{i}} \theta_{i}(t) d t\right)^{-1} .
$$

Applying the inequality between the arithmetic and harmonic means we obtain

$$
\epsilon+\lambda_{2, \theta}(\mathcal{O} ; N) \geq \pi N\left[\frac{1}{N} \sum_{k=1}^{N} N \int_{\delta_{i}} \theta_{i}(t) d t\right]^{-1} \geq \pi N\left[\int_{\mathcal{O}} \theta(t) d t\right]^{-1}
$$

and in view of arbitrariness of $\epsilon>0$ we arrive at the required inequality.

The first part of the previous proof can be carried through unchanged for the cycles. Hence, we have

Lemma 2.5. In the notations of the previous lemma and if $\partial \mathcal{O}_{i}=\emptyset$ for all $1 \leq i \leq s$,

$$
\lambda_{2, \theta}(\mathcal{O})=2 \pi\left[\max _{1 \leq i \leq s} \int_{\mathcal{O}_{i}} \theta(t) d t\right]^{-1} .
$$

As for the higherdimensional case we have only fragmentary facts. Let us assume that $M=\mathbf{R}^{n}$ and $h(x)=|\nabla f| x$ is the euclidean distance from 
the origin. It is clear that $|\nabla f| \nabla 1 h(x)=1$ and $\lambda_{\alpha, 1}(2) \theta \mathcal{O}$ is equal to the ordinary fundamental frequency $\lambda_{\alpha}(\mathcal{O})$. Let $n \geq 2$, then $h$-sphere is the euclidean sphere $S^{n-1}(t)$ of radius $t$, and given open subset $\mathcal{O} \subset S^{n-1}(t)$, we have (see, e.g. [21]),

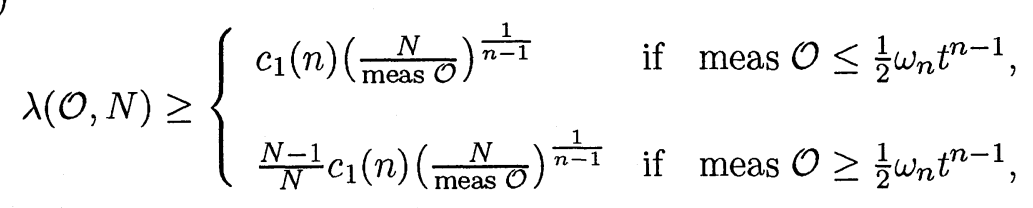

where meas $\mathcal{O}$ is $(n-1)$-dimensional Lebeques measure on $S^{n-1}(t), \omega_{n}=$ meas $S^{n-1}(1)$ and $c_{1}(n)$ depends only on $\mathrm{n}$.

2.4. Let $\mathcal{D} \subset M$ be an open subset and $f \in C^{1}(\mathcal{D})$. For the given $\alpha>1$ we introduce the Dirichlet integral as

$$
\int_{3}|\nabla 2|^{1} \equiv \int_{\mathcal{D}}|\nabla f| \nabla 1 f^{\alpha}
$$

if the last integral is different from the infinity.

Definition 8. Let $P, Q \subset \mathcal{D}$ be disjoint closed sets. We define a capacity of the capacitor $(P, Q ; \mathcal{D})$ by

$$
\operatorname{cap}_{\alpha}(P, Q ; \mathcal{D})=\inf \int_{\mathcal{D}}|\nabla f| \nabla 1 \varphi^{\alpha}
$$

where the infimum is taken over all locally Lipschitz functions $\varphi: M \rightarrow \mathrm{R}^{n} 1$ such that $\varphi(m)=1$ when $m \in P$ and $\varphi(m)=0$ when $m \in Q$.

We say that a manifold $M$ has an $\alpha$-parabolic type if for any compact $F \subset M$ there is a sequence $\mathcal{D}_{1} \subset \mathcal{D}_{2} \subset \ldots \subset \mathcal{D}_{k} \subset \ldots, \cup_{k=1}^{\infty} \mathcal{D}_{k}=M$ of open sets $\mathcal{D}_{k} \supset F$ with compact closures such that

$$
\lim _{k \rightarrow \infty} \operatorname{cap}_{\alpha}\left(F, M \backslash \mathcal{D}_{k} ; M\right)=0
$$

It is easy to see that the last limit doesn't depend on the exhaustion sequence $\left\{\mathcal{D}_{k}\right\}$. Furthermore, we can assume that the family $\left\{\mathcal{D}_{k}\right\}$ is a sequence of $h$-balls for the exhausting function $h(m)$.

Given an $\alpha$-subharmonic function $f(m)$ on $M$, let us denote by $\mathcal{D}=\mathcal{D}(\tau)$ a fixed element of the asymptotic tract $\{\mathcal{D}(\tau)\}$ of $f(m)$. Let $t \in\left(h(\mathcal{D}) ; h_{0}\right)$, where

$$
h(\mathcal{D})=\inf \{h(m): m \in \mathcal{D}\},
$$


and

$$
P(t)=\overline{\mathcal{D}} \cap \overline{B_{h}(t)}, Q(t)=\overline{\mathcal{D}} \backslash B_{h}(t) .
$$

Lemma 2.6. Let $M(t)=\max _{\mathcal{D} \cap \Sigma_{h}(t)}\{\tau, f(m)\}$. Then for any numbers $t_{1}<t_{2}$ from the interval $\left(h(\mathcal{D}), h_{0}\right)$ :

$$
\int_{P\left(t_{1}\right)}|\nabla f| \nabla 1 f^{\alpha} \leq \alpha^{\alpha} \operatorname{cap}_{\alpha}\left(P\left(t_{1}\right), Q\left(t_{2}\right) ; \mathcal{D}\right) M^{\alpha}\left(t_{2}\right) .
$$

Proof. Let $\varphi$ be any function which is admissible for the calculation of $\left(P\left(t_{1}\right), Q\left(t_{2}\right) ; \mathcal{D}\right)$. Then $f_{1}(m)=(f(m)-\tau)$ vanishes everywhere in the set $\partial \mathcal{D} \cap(\operatorname{int} M)$ and $\langle u, e\rangle f_{1}(m) \nu=0$ on $\partial M$. Applying Stokes' formula we obtain

$\int_{\mathcal{D}} \varphi^{\alpha}\langle u, e\rangle \nabla 1 f_{1} \nabla 1 f_{1}|\nabla f| \nabla 1 f_{1}{ }^{\alpha-2} \leq-\alpha \int_{\mathcal{D}} \varphi^{\alpha-1} f_{1}\langle u, e\rangle \nabla 1 f_{1}|\nabla f| \nabla 1 f_{1}{ }^{\alpha-2} \nabla 1 \varphi$.

Hence we have

$$
\int_{\mathcal{D}} \varphi^{\alpha}|\nabla f| \nabla 1 f^{\alpha} \leq \alpha\left(\int_{\mathcal{D}} \varphi^{\alpha}|\nabla f| \nabla 1 f^{\alpha}\right)^{\frac{\alpha-1}{\alpha}}\left(\int_{\mathcal{D}}|\nabla f| f_{1}{ }^{\alpha}|\nabla f| \nabla 1 \varphi^{\alpha}\right)^{\frac{1}{\alpha}} .
$$

and the maximum principle for $f_{1}(m)$ yields

$$
\left(\int_{\mathcal{D}} \varphi^{\alpha}|\nabla f| \nabla 1 f^{\alpha}\right) \leq \alpha^{\alpha} \max _{\mathcal{D} \cap \Sigma_{h}\left(t_{2}\right)} f_{1}^{\alpha}(m) \int_{\mathcal{D} \cap B_{h}\left(t_{2}\right)}|\nabla f| \nabla 1 \varphi^{\alpha} .
$$

Applying now the equality $\varphi(m) \equiv 1$ on $P\left(t_{1}\right)$ and taking infimum over all $\varphi(m)$ we obtain the required estimate and Lemma B.6. is proved.

Lemma 2.7. Let $f(m)$ be an $\alpha$-harmonic function satisfying (1.2) and $M(t)=\max \left\{|\nabla f| f(m): m \in \Sigma_{h}(t)\right\}$. Then for any $t_{1}, t_{2}$ from $\left(0 ; h_{0}\right), t_{1}<$ $t_{2}$ we have

$$
\int_{B_{h}\left(t_{1}\right)}|\nabla f| \nabla 1 f^{\alpha} \leq \alpha^{\alpha}\left[\int_{t_{1}}^{t_{2}} \frac{M^{-\frac{\alpha}{\alpha-1}}(t)}{S\left(\Sigma_{t}\right)} d t\right]^{1-\alpha},
$$

where $S\left(\Sigma_{t}\right)=\left(\int_{\Sigma_{h}(t)}|\nabla f| \nabla 1 h^{\alpha-1}\right)^{\frac{1}{\alpha-1}}$ is the flow of the exhausting function. 
The proof differs only in the details from the same one given above. Namely, from the coarea formula ([7], Theorem 3.2.22) and (2.13) it follows

$$
\int_{B_{h}\left(t_{1}\right)}|\nabla f| \nabla 1 f^{\alpha} \leq \alpha^{\alpha} \int_{B_{h}\left(t_{2}\right)}|\nabla f| f^{\alpha}|\nabla f| \nabla 1 \varphi^{\alpha} \leq \alpha^{\alpha} \int_{t_{1}}^{t_{2}} M^{\alpha}(t) d t \int_{\Sigma_{h}(t)} \frac{|\nabla f| \nabla 1 \varphi^{\alpha}}{|\nabla f| \nabla 1 h}
$$

for any admissible for the capacitor $\left(\overline{B_{h}\left(t_{1}\right)}, M \backslash B_{h}\left(t_{2}\right) ; M\right)$ function $\varphi(m)$

Set

$$
G(t)=\int_{t_{1}}^{t} \frac{d \xi}{M^{\frac{\alpha}{\alpha-1}}(\xi) S(\Sigma(\xi))}
$$

and substitute $G \circ h(m) / G\left(t_{2}\right)$ instead of $\varphi(m)$ into (2.14). Hence, we obtain

$$
\int_{B_{h}\left(t_{1}\right)}|\nabla f| \nabla 1 f^{\alpha} \leq \alpha^{\alpha} G\left(t_{2}\right)^{1-\alpha},
$$

as required.

2.5. Let us suppose that $h(m)$ be a positive $\alpha$-harmonic function everywhere outside of its own null-level set (the main case is $h$ to be the absolute value of a $\alpha$-harmonic function). Then the flow $S\left(\Sigma_{t}\right)$ defined in Lemma ?? does not depend on $t$. The quantity

$$
S(h) \equiv S\left(\Sigma_{t}\right)=\int_{\Sigma_{t}(h)}\langle u, e\rangle \nu \nabla 1 h|\nabla f| \nabla 1 h^{\alpha-2},
$$

is said to be a full flow of $h$.

Lemma 2.8. For any $t_{1}, t_{2}$ from $\left(0, h_{0}\right)$ such that $t_{1}<t_{2}$,

$$
\operatorname{cap}_{\alpha}\left(\overline{B_{h}\left(t_{1}\right)}, M \backslash B_{h}\left(t_{2}\right) ; M\right)=\left(\frac{S(h)}{t_{2}-t_{1}}\right)^{\alpha-1} .
$$

Proof. We notice that the function $\varphi(m)$ defined for $m \in \overline{B_{h}\left(t_{2}\right)} \backslash B_{h}\left(t_{1}\right)$ as

$$
\varphi(m)=\frac{t_{2}-h(m)}{t_{2}-t_{1}}
$$

equal to 0 when $m \in M \backslash B_{h}\left(t_{2}\right)$ and 1 when $m \in B_{h}\left(t_{1}\right)$ is admissible for the capacitor $\left(\overline{B_{h}\left(t_{1}\right)}, M \backslash B_{h}\left(t_{2}\right) ; M\right)$. Then from (2.11) we have

$$
\operatorname{cap}_{\alpha, h}\left(t_{1}, t_{2}\right) \leq \int_{B_{h}\left(t_{2}\right) \backslash \overline{B_{h}\left(t_{1}\right)}}|\nabla f| \nabla 1 \varphi^{\alpha},
$$


where we set

$$
\operatorname{cap}_{\alpha, h}\left(t_{1}, t_{2}\right)=\operatorname{cap}_{\alpha}\left(\overline{B_{h}\left(t_{1}\right)}, M \backslash B_{h}\left(t_{2}\right) ; M\right) .
$$

Applying now $\alpha$-harmonicity of $h(m)$ and the Stokes' formula we obtain

$$
\begin{gathered}
\int_{B_{h}\left(t_{2}\right) \backslash \overline{B_{h}\left(t_{1}\right)}}|\nabla f| \nabla 1 \varphi^{\alpha}=\int_{B_{h}\left(t_{2}\right) \backslash \overline{B_{h}\left(t_{1}\right)}}\left(|\nabla f| \nabla 1 \varphi^{\alpha}+\varphi \operatorname{div}|\nabla f| \nabla \varphi^{\alpha-2} \nabla 1 \varphi\right)= \\
=\int_{\Sigma_{h}\left(t_{2}\right)} \varphi|\nabla f| \nabla 1 \varphi^{\alpha-1}\langle u, e\rangle \nabla 1 \varphi \nu-\int_{\Sigma_{h}\left(t_{1}\right)} \varphi|\nabla f| \nabla 1 \varphi^{\alpha-1}\langle u, e\rangle \nabla 1 \varphi \nu .
\end{gathered}
$$

Now taking into account the boundary conditions on $\varphi(m)$ we arrive at

$$
\operatorname{cap}_{\alpha, h}\left(t_{1}, t_{2}\right) \leq\left(\frac{S(h)}{t_{2}-t_{1}}\right)^{\alpha-1}
$$

To verify that in fact the equality holds, it is sufficient to observe that $\varphi(m)$ is actually an extreme for the calculation of the $\alpha$-capacity of the capacitor $\left(P\left(t_{1}\right), Q\left(t_{2}\right)\right)$, since it is $\alpha$-harmonic and satisfies the "natural" boundary condition $\langle u, e\rangle \nabla 1 h \nu=0$ on $\partial M$ (see e.g. [6]).

\section{The Main Inequality.}

3.1. Let $M$ be a noncompact $p$-dimensional manifold with a fixed exhausting function $h(m): M \rightarrow\left[0, h_{0}\right)$. We assume that $f$ is an $\alpha$ subharmonic function satisfying $(1.2)$ and $\{\mathcal{D}(\tau)\}$ is an asymptotic tract of $f$. Let us choose an arbitrary subset $\mathcal{D} \equiv \mathcal{D}\left(\tau_{0}\right)$, where $\tau_{0}$ is a regular value of both $h$ and $f$, and set $f_{1}(m)=f(m)-\tau_{0}$ everywhere in $\mathcal{D}$. Let $h(\mathcal{D})=\inf _{m \in \mathcal{D}} h(m)$. Then for any regular value $t>h(\mathcal{D})$ of $h(m)$ we have

$$
J(t) \equiv \int_{3}|\nabla 2|^{1}=\int_{\mathcal{D} \cap B_{h}(t)}|\nabla f|^{\alpha} \leq \int_{\mathcal{D} \cap \Sigma_{h}(t)} f_{1}\langle u, e\rangle \nabla f_{1}\left|\nabla f_{1}\right|^{\alpha-2} \frac{\nabla h}{|\nabla h|},
$$

because $f_{1}(m) \Delta_{\alpha} f_{1}(m) \geq 0$ everywhere in $\mathcal{D}$. Here $\nabla h /|\nabla h|$ is the outward normal $\nu(m)$ to $\Sigma_{h}(t) \cap \mathcal{D}$ with respect to $M$. The set $\Sigma_{h}(t) \cap \mathcal{D}$ is compact and, hence, it splits into the cycles $\Gamma_{1}(t), \ldots, \Gamma_{l}(t)$ and components $\gamma_{1}(t), \ldots, \gamma_{k}(t)$ with nonempty boundaries. Let $\Gamma(t)=\cup_{i=1}^{l} \Gamma_{i}(t)$ and $\gamma(t)=\cup_{j=1}^{k} \gamma_{j}(t)$. We set

$$
\lambda(t)=\min \left\{\lambda_{\alpha, \nabla h}\left(\gamma_{i}\right), \lambda_{\alpha, h}\left(\Gamma_{j}\right)\right\}
$$


and $q_{j}(t)=\phi_{\alpha}\left(f_{1}(m) ; \Gamma_{j}(t)\right)$. Then the function

$$
v(m)= \begin{cases}f_{1}(m), & m \in \gamma(t) \\ f_{1}(m)-q_{j}(t), & m \in \Gamma_{j}(t) \text { for } 1 \leq j \leq l\end{cases}
$$

is admissible for $\Sigma_{h}(t) \cap \mathcal{D}$. Applying the Minkowski inequality we have

$$
\begin{gathered}
v\langle u, e\rangle \nabla f_{1}\left|\nabla f_{1}\right|^{\alpha-2} \nabla h \leq\left.\left.\frac{\alpha-1}{\lambda(t) \alpha}\left|\langle u, e\rangle \nabla f_{1}\right| \nabla f_{1}\right|^{\alpha-2} \frac{\nabla h}{|\nabla h|}\right|^{\frac{\alpha}{\alpha-1}}+ \\
\frac{1}{\alpha} \lambda^{\alpha-1}(t)|v|^{\alpha}|\nabla h|^{\alpha},
\end{gathered}
$$

and, therefore,

$$
\begin{gathered}
J(t) \leq \int_{\Sigma_{h}(t) \cap \mathcal{D}} v\langle u, e\rangle \nabla f_{1}\left|\nabla f_{1}\right|^{\alpha-2} \frac{\nabla h}{|\nabla h|}+\sum_{j=1}^{l} q_{j}(t) \int_{\Gamma_{j}}\langle u, e\rangle \nabla f_{1}\left|\nabla f_{1}\right|^{\alpha-2} \frac{\nabla h}{|\nabla h|} \leq \\
\frac{\lambda^{\alpha-1}(t)}{\alpha} \int_{\Sigma_{h}(t) \cap \mathcal{D}}|v|^{\alpha}|\nabla h|^{\alpha-1}+ \\
\left.\left.\frac{\alpha-1}{\alpha \lambda(t)} \int_{\Sigma_{h}(t) \cap \mathcal{D}} \frac{1}{|\nabla h|}\left|\langle u, e\rangle \nabla f_{1}\right| \nabla f_{1}\right|^{\alpha-2} \frac{\nabla h}{|\nabla h|}\right|^{\frac{\alpha}{\alpha-1}}+Q(t),
\end{gathered}
$$

where

$$
Q(t)=\sum_{j=1}^{l} q_{j}(t) \int_{\Gamma_{j}(t)}\langle u, e\rangle \nabla f_{1}\left|\nabla f_{1}\right|^{\alpha-2} \nu .
$$

Taking into account the definition of $\lambda(t)$ we arrive at

$$
J(t) \leq \frac{1}{\alpha \lambda(t)} \int_{\Sigma_{h}(t) \cap \mathcal{D}} \frac{|D v|^{\alpha}}{|\nabla h|}+
$$

$$
\frac{\alpha-1}{\alpha \lambda(t)} \int_{\Sigma_{h}(t) \cap \mathcal{D}} \frac{\left|\nabla f_{1}\right|^{\alpha}}{|\nabla h|}\left|\left\langle\frac{\nabla f_{1}}{\left|\nabla f_{1}\right|}, \frac{\nabla h}{|\nabla h|}\right\rangle\right|^{\frac{\alpha}{\alpha-1}}+Q(t),
$$


where $D$ is the induced covariant derivative on $\Sigma(t)$.

By virtue of orthogonality of $\nu=\nabla h(m)|\nabla h(m)|^{-1}$ to the tangent space $T_{m} \Sigma_{h}(t)$, we obtain the following gradient decomposition formula

$$
\nabla f_{1}=D f_{1}+\nu\langle u, e\rangle \nabla f_{1} \nu .
$$

Moreover, $D v=D f_{1}$ everywhere in $\mathcal{D} \cap \Sigma_{h}(t)$. Hence, from (3.2), (3.1) and (3.3) we obtain

$$
J(t) \leq Q(t)+\frac{1}{\lambda(t)} \int_{\Sigma_{h}(t) \cap \mathcal{D}} \frac{\left|\nabla f_{1}\right|^{\alpha}}{|\nabla h|}\left(\frac{\xi^{\alpha}}{\alpha}+\frac{\alpha-1}{\alpha}\left(1-\xi^{2}\right)^{\frac{\alpha}{2(\alpha-1)}}\right),
$$

where $\xi=\left|D f_{1}\right| /\left|\nabla f_{1}\right| \leq 1$. Analysis of the last expression in the parentheses shows that it is decreasing function on $\xi$ for $\alpha \geq 2$ and increasing one if $1<\alpha<2$. Hence,

$$
J(t) \leq Q(t)+\frac{c(\alpha)}{\lambda(t)} \int_{\Sigma_{h}(t) \cap \mathcal{D}} \frac{\left|\nabla f_{1}\right|^{\alpha}}{|\nabla h|}
$$

where

$$
c(\alpha)=\left\{\begin{array}{l}
\frac{\alpha-1}{\alpha} \text { for } \alpha \geq 2 \\
\frac{1}{\alpha} \text { for } \alpha \in(1 ; 2) .
\end{array}\right.
$$

Because the set $\Sigma_{h}(t) \cap \mathcal{D}$ is the $t$-level of $h$, the coarea formula yields

$$
J(t) \leq Q(t)+\frac{c(\alpha)}{\lambda(t)} \frac{d J}{d t}
$$

for a.e. $t \in\left(h(\mathcal{D}), h_{0}\right)$.

3.2. We assume in this paragraph that there exists at least one regular asymptotic tract of $f$. Let $\Delta$ be the open subset of $\left(h(\mathcal{D}) ; h_{0}\right)$ corresponding to regularity of $\mathcal{D}\left(\tau_{0}\right)$, i.e. $\Gamma(t)=\emptyset$ for $t \in \Delta$, where

$$
\Delta=\cup_{i=1}^{\infty} \Delta_{i}, \quad \Delta_{i}=\left(\alpha_{i}, \beta_{i}\right),
$$


and $h_{0} \in \bar{\Delta}$. We observe that the characteristic $J(t)$ is nondecreasing function. Moreover, for $t \in \Delta$ we have by Lemma ?? that $\lambda(t)=\lambda_{\alpha, h}^{\star}\left(\Sigma_{h}(t) \cap \mathcal{D}\right)$, and by virtue of (3.4) we obtain

$$
J^{\prime}(t) \geq c(\alpha) \lambda_{\alpha, h}^{\star}\left(\Sigma_{h}(t) \cap \mathcal{D}\right) J(t)
$$

almost everywhere in $\left(h(\mathcal{D}), h_{0}\right)$. By virtue of absolute continuity of $J(t)$, we arrive at

Theorem 3.1. Let $f(m)$ be an $\alpha$-subharmonic function on $M$ and $\mathcal{D}=$ $\mathcal{D}\left(\tau_{0}\right)$ be a regular component of an asymptotic tract $\{\mathcal{D}(\tau)\}$. Then for any $t_{1}, t_{2}$ from $\left(h(\mathcal{D}), h_{0}\right)$

$$
\int_{3}|\nabla 2|^{1} \leq \int_{3}|\nabla 2|^{1} \exp \left(-c(\alpha) \int_{\left(t_{1}, t_{2}\right) \cap \Delta} \lambda_{\alpha, h}^{\star}\left(\Sigma_{h}(t) \cap \mathcal{D}\right) d t\right) .
$$

Corollary 3.2. Let $f$ be an $\alpha$-subharmonic function having at least $N$ different regular asymptotic tracts $\left\{\mathcal{D}_{i}(\tau)\right\}, \ldots,\left\{\mathcal{D}_{N}(\tau)\right\}$ and $\sigma=$ $\max _{1 \leq i \leq N} h\left(\mathcal{D}_{i}\right)$, where $\mathcal{D}_{i} \equiv \mathcal{D}_{i}\left(\tau_{i}\right)$ are regular elements. Then for any $t_{1}<t_{2}$ from $\left(\sigma, h_{0}\right)$

$$
N \min _{1 \leq i \leq N} \int_{3}|\nabla 2|^{1} \leq \int_{3}|\nabla 2|^{1} \exp \left(-c(\alpha) \int_{t_{1}}^{t_{2}} \lambda_{\alpha, h}^{\star}\left(\Sigma_{h}(t) ; N\right) d t\right)
$$

Proof. According to the previous theorem we have for every domain $\mathcal{D}_{i}$ and for $t_{1}<t_{2}, t_{i} \in\left(\sigma, h_{0}\right)$ that

$$
\exp \left(c(\alpha) \int_{t_{1}}^{t_{2}} \lambda_{i}(t) d t\right) \int_{B_{h}\left(t_{1}\right) \cap \mathcal{D}_{i}}|\nabla f|^{\alpha} \leq \int_{\mathcal{D}_{i} \cap B_{h}\left(t_{2}\right)}|\nabla f| \nabla 1 f^{\alpha},
$$

where $\lambda_{i}(t)=\lambda_{\alpha, h}^{\star}\left(\Sigma_{h}(t) \cap \mathcal{D}_{i}\right)$. Summing the last inequalities yields

$$
\min _{1 \leq i \leq N} \int_{3}|\nabla 2|^{1} \cdot \sum_{i=1}^{N} \exp \left(c(\alpha) \int_{t_{1}}^{t_{2}} \lambda_{i}(t) d t\right) \leq \int_{3}|\nabla 2|^{1}
$$


Applying the inequality between arithmetic and geometric means in the left side of (3.6) we arrive at

$$
\min _{1 \leq i \leq N} \int_{3}|\nabla 2|^{1} \cdot N \exp \frac{1}{N} \sum_{i=1}^{N}\left(c(\alpha) \int_{t_{1}}^{t_{2}} \lambda_{i}(t) d t\right) \leq \int_{3}|\nabla 2|^{1} .
$$

But the domains $\mathcal{D}_{1}, \ldots, \mathcal{D}_{N}$ are pairwise nonoverlapping ones, and therefore for every $t \in\left(t_{1}, t_{2}\right)$

$$
\frac{1}{N} \sum_{i=1}^{N} \lambda_{i}(t)=\frac{1}{N} \sum_{i=1}^{N} \lambda_{\alpha, h}^{\star}\left(\mathcal{D}_{i} \cap \Sigma_{h}(t)\right) \geq \lambda_{\alpha, h}\left(\Sigma_{h}(t), N\right) .
$$

Hence, the required inequality is proved.

Corollary 3.3. Let $M$ be a manifold with an exhausting function $h(m)$ such that for some integer $N \geq 1$ and $h_{1}<h_{0}$

$$
\int_{h_{1}}^{h_{0}} \lambda_{\alpha, h}\left(\Sigma_{h}(t) ; N\right) d t=+\infty .
$$

Then every $\alpha$-subharmonic function $f(m)$ with finite Dirichlet integral

$$
\int_{M}|\nabla f|^{\alpha}<\infty
$$

have at most $(N-1)$ different regular asymptotic tracts.

Proof. Really, if $f(m)$ has $N$ different regular asymptotic tracts, then from (3.5) and (3.7) by finiteness of the Dirichlet integral we conclude for some $i \leq N$ and $t_{i} \in\left(h\left(\mathcal{D}_{i}\right), h_{0}\right)$ that everywhere in the open set $\mathcal{D}_{i} \cap B_{h}\left(t_{i}\right)$ one holds $|\nabla f| \equiv 0$. It follows that $f(m) \equiv$ const when $m \in \mathcal{D}_{i} \cap B_{h}\left(t_{i}\right)$, that contradicts the definition of $\mathcal{D}_{i}=\mathcal{D}_{i}\left(\tau_{i}\right)$.

Corollary 3.4. Let $M$ be a manifold without a boundary and an exhausting function $h$ satisfies

$$
\int_{h_{1}}^{h_{0}} \lambda_{\alpha, h}\left(\Sigma_{h}(t) ; 2\right) d t=+\infty
$$


Then constants are only $\alpha$-harmonic functions on $M$ with finite Dirichlet integral.

Proof. Let $\Delta=\cup_{i} \Delta_{i}$ be the subset of $\left(h_{1}, h_{0}\right)$ consisting of the regular values $t$ of $h$ such that $\lambda_{\alpha, h}\left(\Sigma_{h}(t), 2\right)>0$. It follows from Remark 1 that $\Sigma_{h}(t)$ can contain one cyclic components at most. On the other hand, $\Sigma_{h}\left(t_{0}\right)$ is a compact submanifold without a boundary for all $t_{0} \in \Delta$. Hence, if $\Sigma_{h}\left(t_{0}\right)$ contains a cyclic component then it must contain two or more such components. But this contradicts with $t_{0} \in \Delta$ and shows that $\Sigma_{h}\left(t_{0}\right)$ hasn't any cyclics.

Now, let $f(m)$ be an arbitrary $\alpha$-harmonic function different from a constant. Given a fixed point $m_{0}$ we consider the new subharmonic function $f_{1}(m)=\left|f(m)-f\left(m_{0}\right)\right|$ which has at least two different asymptotic tracts. The last property is a direct consequence of maximum principle for $f_{1}$. Then it follows from the arguments above that these tracts are regular.

Other conditions concerning the Liouville type theorems can be found, for instance, in [8], [9]. We emphasize that our assertions have been formulated without any requirements on the geodesic completeness. The following example shows that the conditions (3.7), (3.8) do not yield it.

Example 5. Let $M$ be realized as the compact rotational hypersurface in $\mathbf{R}^{n}$ with a profile function $\rho\left(x_{n}\right)$, i.e.

$$
x_{1}^{2}+x_{2}^{2}+\ldots+x_{n-1}^{2}=\rho^{2}\left(x_{n}\right), \quad x_{n}>0 .
$$

We suppose that $\rho(0)=0$ and the original in $\mathbf{R}^{n}$ is the limit singular point of $M$. Namely,

$$
\rho(t)=\left\{\begin{aligned}
t & \text { for } t \in(0 ; 1) \\
\zeta(t) & \text { for } t \in(1 ; 2) \\
\sqrt{9-t^{2}} & \text { for } t \in(2 ; 3)
\end{aligned}\right.
$$

where $\zeta(t)$ is a sufficient smooth sewing function. Thus $M$ is a "drop" with regular top point $A=(0, \ldots, 0,3)$. Then the geodesic distance $h(m)$ from $A$ to $m \in M$ is an exhausting function on $M$ and

$$
h_{0}=\lim _{m \rightarrow 0} h(m)<+\infty .
$$

Therefore, $M$ isn't geodesically complete. On the other hand, all $h$-spheres $\Sigma_{h}(t)$ are $(n-2)$-dimensional Euclidean spheres of radius $R(t)$. Here $R(t)=$ 
$\rho(m)$ where $h(m)=t$. It follows for the values of $h(m)$ sufficiently closed to $h_{0}$ that

$$
R(h(m))=\frac{1}{\sqrt{2}}\left(h_{0}-h(m)\right) .
$$

We observe also that $|\nabla h| \equiv 1$ everywhere in $M \backslash\{A\}$. Hence, from (2.10) we obtain

$$
\lambda_{\alpha, h}\left(\Sigma_{h}(t), N\right)=\frac{c}{h_{0}-t}, \text { as } t \rightarrow h_{0},
$$

where $c$ depends only on $n, N$. We see that (3.8) is valid, while $M$ isn't geodesic complete.

3.3. Now we suppose that an $\alpha$-subharmonic function $f(m)$ has at least $N \geq 1$ regular asymptotic tracts $\left\{\mathcal{D}_{1}(\tau)\right\}, \ldots,\left\{\mathcal{D}_{N}(\tau)\right\}$ and choose pairwise disjoint domains $\mathcal{D}_{k}=\mathcal{D}_{k}\left(\tau_{k}\right), 1 \leq k \leq N$ such that the sets $\mathcal{D}_{k} \cap \Sigma_{h}(t)$ would answer to the regularity's condition.

Applying (2.12) and (3.6) we obtain

$$
\begin{gathered}
\min _{1 \leq i \leq N} \int_{3}|\nabla 2|^{1} \sum_{k=1}^{N} \exp \left\{c(\alpha) \int_{t_{1}}^{t_{2}} \lambda_{\alpha, h}^{\star}\left(\mathcal{D}_{k} \cap \Sigma_{h}(t)\right) d t\right\} \leq \\
\alpha^{\alpha} M^{\alpha}\left(t_{3}\right) \operatorname{cap}_{\alpha, h}\left(t_{2}, t_{3}\right) .
\end{gathered}
$$

where $M(t)=\max _{m \in \Sigma_{h}(t)}\left\{\tau_{1}, \tau_{2}, \ldots, \tau_{N}, f(m)\right\}$ and the numbers $t_{1}<t_{2}<$ $t_{3}$ belong to $\left(h_{1}, h_{0}\right)$.

Arguing as in the proof of the corollary C.2. we conclude

$$
\begin{gathered}
\min _{1 \leq i \leq N} \int_{3}|\nabla 2|^{1} \leq \\
\frac{\alpha^{\alpha} M^{\alpha}\left(t_{3}\right)}{N} \operatorname{cap}_{\alpha, h}\left(t_{2}, t_{3}\right) \exp \left(-c(\alpha) \int_{t_{1}}^{t_{2}} \lambda_{\alpha, h}\left(\Sigma_{h}(t), N\right) d t\right) .
\end{gathered}
$$

Thus, we have proved the following version of the Denjoy-Ahlfors theorem.

Theorem 3.5. Let $f(m)$ be an $\alpha$-subharmonic function and for some integer $N \geq 1$

$$
\liminf _{t, \xi \rightarrow h_{0}} M(\xi) \operatorname{cap}_{\alpha, h}^{\frac{1}{\alpha}}(t, \xi) \exp \left(-\frac{c(\alpha)}{\alpha} \int_{t_{1}}^{t} \lambda_{\alpha, h}\left(\Sigma_{h}(t), N\right) d t\right)=0,
$$


where $M(t)=\max _{\Sigma_{h}(t)} f^{+}(m), t_{1}>0$ is fixed and $t, \xi$ are tending to $h_{0}$ such that $t_{1}<t<\xi$. Then $f(m)$ has at most $(N-1)$ different regular asymptotic tracts.

4. The cyclic case: singular asymptotic tracts.

4.1. We observe (see for a detailes.[13], [21]) that the topologically trivial manifolds such as $\mathbf{R}^{n}$ or graphs over $\mathbf{R}^{n}$ admit a big store of subharmonic functions with regular asymptotic tracts. On the other hand, there exist manifolds having nontrivial harmonic functions with compact level-sets. This means that for such a manifold the term $\lambda\left(\Sigma_{h}(t) ; N\right)$ is trivial. It is a consequence of the definition of this value by the reduced fundamental frequency. To deal with such manifolds we must refine our tool. On the other hand, there are some difficulties in the higherdimensional case. Namely, we need the lower estimations of the first eigenvalue of compact manifolds. In this section we consider the two-dimensional case only and will assume that $\alpha=2$.

Definition 9. One said that a manifold $M$ admits the harmonic exhausting if there exists an exhausting function $h(m)$ of $M$ satisfying the property: $\Delta h(m)=0$ everywhere outside of $\Sigma_{h}(0)$. If $\partial M$ is not empty we'll assume that the condition (1.2) holds for $h$ also.

Let $f(m)$ be a harmonic function on $M$ and $t_{0}$ be a regular value of $h(m)$. Then the characteristic $Q(t)$ introduced in (3.1) is differentiable at $t_{0}$. Really, it is a consequence of the Stokes formula and the property of orthogonality of $\nabla h$ to $\Gamma_{j}(t)$ that 


$$
\begin{aligned}
\frac{d q_{j}\left(t_{0}\right)}{d t} & =\frac{d}{d t}\left[S^{-i 1}\left(h, \Gamma_{j j}\left(t_{0}\right)\right) \int^{\Gamma_{j}(t)}\|\nabla h\| f\right]_{t=t_{0}}\left[\frac{d}{d t} \int^{\Gamma_{j}(t)} f\langle\nabla h, \nu\rangle\right]_{t=t_{0}} \\
& =\frac{1}{S\left(h, \Gamma_{j}\left(t_{0}\right)\right)}\left[\int^{\Gamma_{j}\left(t_{0}\right)} \frac{1}{\operatorname{div}(f \nabla h)}\right. \\
& =\frac{1 \nabla h \|}{S\left(h, \Gamma_{j}\left(t_{0}\right)\right)} \\
& =\frac{1}{S\left(h, \Gamma_{j}\left(t_{0}\right)\right)} \int^{\Gamma_{j}\left(t_{0}\right)}\left\langle\nabla f, \frac{\nabla h}{\|\nabla h\|}\right\rangle \\
& =\frac{S\left(f, \Gamma_{j}\left(t_{0}\right)\right)}{S\left(h, \Gamma_{j}\left(t_{0}\right)\right)} .
\end{aligned}
$$

Here by $S(\varphi, \Gamma)$ we denote the flow of $\varphi$ through $\Gamma$. Now from (4.1) and (3.1) we obtain

$$
\frac{d Q}{d t}\left(t_{0}\right)=\sum_{j=1}^{l} \frac{S^{2}\left(f, \Gamma_{j}\left(t_{0}\right)\right)}{S\left(h, \Gamma_{j}\left(t_{0}\right)\right)} .
$$

From harmonicity of $f$ and $h$ we obtain the following

Proposition 4.1. The derivative $\omega(t)=Q^{\prime}(t)$ is a piecewise constant function. Moreover, $Q(t)$ is a piecewise linear function.

We observe that the full flows of $f$ and $h$ through $\Gamma(t)$ does not depend on the value $t$. Moreover,

$$
S(h)=S(h, \Gamma(t))=\sum_{j=1}^{l} S\left(h, \Gamma_{j}(t)\right)=\int_{\Gamma(t)}|\nabla h|>0
$$

and from Cauchy's inequality we have

$$
\omega(t)=\frac{d Q}{d t} \geq \frac{S^{2}(f)}{S(h)}
$$

4.2. In this paragraph we suppose that $h(m)$ has isolated critical points only. We notice that this requirement is realized when $\operatorname{dim} M=2$. Really, due M.Morse, every harmonic function $f(m)$ on $M$ is simultaneously a 
pseudoharmonic function in local coordinates of $M$, and the needed assertion follows from the standard properties of pseudoharmonic functions.

It follows that $Q(t)$ is continuous and hence

$$
Q(t)=\int_{0}^{t} \omega(\eta) d \eta
$$

Hence, the Main Inequality provides the following relation

$$
\frac{d J(t)}{d t} \geq 2 \lambda_{h}\left(\Sigma_{h}(t)\right)\left(J(t)-\int_{0}^{t} \omega(\eta) d \eta\right)
$$

where

$$
J(t)=\int_{B_{h}(t)}|\nabla f|^{2} .
$$

We need the following consequence of Lemma B.5.:

Proposition 4.2. Let $M$ be a two-dimensional manifold and $h(m)$ be a harmonic exhausting on $M$. Then

$$
\lambda_{h}\left(\Sigma_{h}(t)\right)=2 \pi \min _{1 \leq j \leq l}\left\{S^{-1}\left(h, \Gamma_{j}(t)\right)\right\} \geq 4 \pi S^{-1}(h) .
$$

Proof. We notice by harmonicity of $h(m)$ that the set $\Sigma_{h}(t)$ splits into the union of two nonempty level-sets $\Sigma_{h}^{ \pm}(t)=\{m: h(m)= \pm t\}$. Moreover, by the Stokes' formula we have

$$
S\left(h, \Sigma_{h}^{+}(t)\right)=S\left(h, \Sigma_{h}^{-}(t)\right)=\frac{1}{2} S(h),
$$

and positivity of the flow yields

$$
S\left(h, \Gamma_{j}(t)\right) \leq S\left(h, \Sigma_{h}^{ \pm}(t)\right)=\frac{1}{2} S(h) .
$$

Hence (4.3) follows from (2.9) and (4.4). 
Let $e$ be a fixed unit vector in $\mathbf{R}^{n}$ and $\left\{\Pi_{t}\right\}$ be the family of all hyperplanes given by the equation $\langle u, e\rangle x e=t$. We notice that every $\Pi_{t}$ is orthogonal to $e$.

Definition 10. ([26]) A surface $\mathcal{M}=(M, x)$ is called tubular if there exists an interval $(a ; b)$ such that any portion of $\mathcal{M}$ situated between two hyperplanes $\Pi_{t_{1}}$ and $\Pi_{t_{2}}$ with $t_{i} \in(a ; b)$ is compact and any section $\Pi_{t} \cap \mathcal{M}$ is not empty. If $(a ; b)$ coincide with the whole $\mathbf{R}^{n} 1$ we call $\mathcal{M}$ to be tubular in the large.

The simplest example of a tubular in the large minimal surface in $\mathbf{R}^{n} 3$ is the catenoid. Numerous examples of two-dimensional immersed tubular minimal surfaces in $\mathbf{R}^{n}$ can be constructed by the representation for such surfaces given in [29]. It follows from the papers [26], [25], [16] that all $p$-dimensional tubular minimal surfaces of arbitrary codimension and $p \geq 3$ are bounded in the $e$-direction, i.e. they can not be tubular in the large.

To simplify the argumens we shall assume that $a=-b$. The general case can be reduced to this one with the suitable shift of $\mathcal{M}$ along the $e$-axis. We set $h(m)=|\langle u, e\rangle x(m) e|$ to be exhausting function on $M$ provided that $\mathcal{M}=(M ; x)$ be a minimal surface. Moreover, $\Delta h(m)=0$ everywhere in $M \backslash \Pi_{0}$ and hence, $h(m)$ is a harmonic exausting.

Theorem 4.3. Let $\mathcal{M}$ be a two-dimensional tubular with respect to e minimal surface in $\mathbf{R}^{n}$. Let $f$ be a harmonic function on $M$ with the Dirichlet integral $J(t)$. If

$$
\lim _{t \rightarrow b-0} \frac{1}{J(t)} \int_{0}^{t} \omega(y) d y=0,
$$

then

$$
\liminf _{t \rightarrow b-0} \frac{\ln J(t)}{t} \geq \frac{8 \pi}{S(h)} .
$$

Proof. We rewrite (4.2) with applying of Proposition D.2. as following

$$
J^{\prime}(t) \geq \frac{8 \pi}{S(h)}[J(t)-Q(t)]
$$

or,

$$
\frac{d}{d t}[J(t) \exp (-8 \pi t / S(h))] \geq-\frac{8 \pi Q(t)}{S(h)} \exp (-8 \pi t / S(h)) .
$$

After integrating, we obtain 


$$
J(t) \exp (-8 \pi t / S(h)) \geq-\int_{0}^{t} Q(y) \exp (-8 \pi y / S(h)) d y
$$

Now (4.5) yields the needed assertion.

\section{Some applications to the theory of minimal and $\alpha$-minimal surfaces.}

5.1. Let $M$ be an orientiable noncompact $p$-dimensional manifold and $x: M \rightarrow \mathrm{R}^{n}$ be a smooth isometric immersion. Let $\nu$ be the unit normal vector field to $\mathcal{M}=(M, x)$ and $A^{\nu}$ be the corresponding Weingarten map.

The quantity $k_{\nu}(E)=\langle u, e\rangle A^{\nu} E E$ is called the curvature in $E \in T_{m} M$ direction.

We have already used the following characterestic property of the minimal surfaces. Namely, they are only surfaces in $\mathbf{R}^{n}$ with harmonic coordinate functions. On the other hand, let $\Delta_{\alpha}$ be the $\alpha$-Laplacian of $M$ associated with the induced metric. We fix a vector $e$ in $\mathbf{R}^{n} n+1$ and denote by $\tau=e^{\top} /|\nabla f| e^{\top}$ the tangent to $\mathcal{M}$ direction of the largest increasing of the coordinate function $f_{e}(m)=\langle u, e\rangle e x(m)$.

Definition 11. [36] We say that a surface $\mathcal{M}$ is $\alpha$-minimal if its mean curvature $h(m)$ and the curvature $k_{\nu}(\tau)$ satisfy the following linear relation

$$
h(m)=-(\alpha-2) k_{\nu}(\tau),
$$

at every point $m$ such that $e^{\top}(m) \neq 0$.

The definition above is motived by the following usefull analytic interpretation of condition (5.1).

Proposition 5.1. A hypersurface $\mathcal{M}$ is $\alpha$-minimal if and only if the coordinate function $f(m)$ is $\alpha$-harmonic in the inner metric of $\mathcal{M}$.

Proof. We have $\nabla 1 f=e^{\top}$ for the gradient of $f(m) \equiv f_{e}(m)$ and for a tangent vector $X$ we obtain

$\nabla_{X}|\nabla f| \nabla 1 f=\nabla_{X}|\nabla f| e^{\top}=\frac{\langle u, e\rangle \bar{\nabla} 1_{X} e^{\top} e^{\top}}{|\nabla f| e^{\top}}=\frac{\langle u, e\rangle-\bar{\nabla} 1_{X} e^{\perp} e^{\top}}{|\nabla f| e^{\top}}=\frac{\langle u, e\rangle A^{e^{\perp}} X e^{\top}}{|\nabla f| e^{\top}}$.

By self-conjugasy of the Weingarten map $A^{e^{\perp}}$ we conclude

$$
\nabla 1|\nabla f| \nabla 1 f=A^{e^{\perp}}(\tau)
$$


everywhere in $\left\{m:|\nabla 1 f| \equiv|\nabla f| e^{\top} \neq 0\right\}$. Thus,

$$
\begin{gathered}
\Delta_{\alpha} f=\operatorname{div}|\nabla f| \nabla 1 f^{\alpha-2} \nabla 1 f=|\nabla f| \nabla 1 f^{\alpha-2} \Delta f+(\alpha-2)\langle u, e\rangle \nabla|\nabla f| \nabla 1 f \nabla 1 f|\nabla f| \nabla 1 f^{\alpha-3}= \\
=|\nabla f| \nabla 1 f^{\alpha-4}\left[|\nabla f| e^{\top^{2}} \Delta f+(\alpha-2)\langle u, e\rangle A^{e^{\perp}}\left(e^{\top}\right) e^{\top}\right]
\end{gathered}
$$

and applying the known [17] connection between the Laplacian and the mean curvature

$$
\Delta_{\alpha} f=|\nabla f| e^{T^{\alpha-2}}\langle u, e\rangle H+(\alpha-2) B(\tau, \tau) e^{\perp} .
$$

If $\mathcal{M}$ is a hypersurface then $e^{\perp}=\langle u, e\rangle e \nu \nu$ and the definition of $\alpha$ harmonicity of $f(m)$ can be written as

$$
\left(h(m)+(\alpha-2) k_{\nu}(\tau)\right)|\nabla f| e^{\top \alpha-2}=0,
$$

which proves the Proposition E.1.

5.2. We remind that for two smooth manifolds $M_{1}$ and $M_{2}$ a mapping $F: M_{1} \rightarrow M_{2}$ is called the mapping with bounded distortion, or quasiregular mapping, if the Jacobian $\operatorname{det} d_{x} F$ doesn't change the sign on $M_{1}$ and there exists a constant $K \geq 1$ such that

$$
\max \left|d_{x} F(E)\right| \leq K \min \left|d_{x} F(E)\right|, \quad x \in M,
$$

where the minimum and maximum are taken over all unit tangent vectors $E \in T_{x} M_{1}$. The smallest constant $K \equiv K(F)$ is called the distortion coefficient of $F(x)$ ([1], [31]).

Another well-known fact (see [30]) is that the Gauss map

$$
\gamma: M \rightarrow S^{2}
$$

of two-dimensional minimal surface into the standart sphere is conformal. The following assertion was announced in [36] and extends that property on the class of $\alpha$-minimal surfaces.

Theorem 5.2. Let $\alpha>1$. Then the Gauss map $\gamma$ of a two-dimensional $\alpha$-minimal surface is a mapping with bounded distortion. Moreover,

$$
K(\gamma) \leq \max \left(\alpha-1 ; \frac{1}{\alpha-1}\right) .
$$


Proof. We observe that the tangent spaces $T_{m} M$ to $M$ and $T_{\gamma(m)} S^{2}$ to sphere $S^{2}$ can regarded as canonically isomorphic ones. Really, we identify the image of the Weingarten map $A(E)$ with $d \gamma_{m}(E)$.

Let us consider an arbitrary point $m$ such that $e^{\top}(m) \neq 0$ and choose the orthonormal basis $E_{1}, E_{2}$ of the tangent space at $m$ which one diagonalizes the symmetric Weingarten map $A^{\nu}$ :

$$
A^{\nu}\left(E_{i}\right)=\lambda_{i} E_{i}
$$

where $\lambda_{1}, \lambda_{2}$ are the principal curvatures of $\mathcal{M}$ at $m$. Set $\tau=e^{\top} /\left|e^{\top}\right|$. Then for some angle $\psi \in[0 ; 2 \pi)$ we have

$$
\tau=E_{1} \cos \psi+E_{2} \sin \psi
$$

and by virtue of (5.1),

$$
\langle u, e\rangle A^{\nu}(\tau) \tau=\lambda_{1} \cos ^{2} \psi+\lambda_{2} \sin ^{2} \psi=-\frac{1}{\alpha-2}\left(\lambda_{1}+\lambda_{2}\right) .
$$

Thus,

$$
\lambda_{1}=-\lambda_{2} \frac{1+(\alpha-2) \sin ^{2} \psi}{1+(\alpha-2) \cos ^{2} \psi} .
$$

We observe that the last identity yields negativity of Jacobian $\operatorname{det}\left(d_{m} \gamma\right)=\lambda_{1} \lambda_{2}$ at $m$. Now, using the extremal properties of the quadratic forms we obtain the value of the distortion coefficient $K$ at $m$,

$$
K_{m}=\max _{\psi}\left\{q ; \frac{1}{q}\right\}, \quad q=\frac{1+(\alpha-2) \sin ^{2} \psi}{1+(\alpha-2) \cos ^{2} \psi} .
$$

An easy computation of the maximal value of the right part over all admissible angles $\psi$ yields the required inequality.

If $e^{\top}(m)=0$, then one can show (see [36]) that Weingarten map $A^{\nu}$ is the identical null and (5.2) is trivial.

Corollary 5.3. For $\alpha>1$ two-dimensional planes are only entire $\alpha$ minimal graphs in $\mathbf{R}^{n} 3$.

Proof. This version of the well-known S.N.Bernstein's result is a direct consequence of the previous theorem and the theorem of L.Simon [32] on the two-dimensional entire graphs with quasiconformal Gauss map. 
5.3. Let $\alpha=2, M$ be a two-dimensional manifold and $f(m)$ be a subharmonic function on $M$ having $N$ different regular asymptotic tracts $\mathcal{D}_{1}, \mathcal{D}_{2}, \ldots, \mathcal{D}_{N}$. Let $h(m)$ be an exhausting function on $M$ and

$$
\theta(t)=\int_{\Sigma_{h}(t)}|\nabla f| \nabla 1 h
$$

is the flow of $h(m)$ through $\Sigma_{h}(t)$. Then we have from Lemma ?? and inequalities (2.9), (3.5) for any $t_{1}<t_{2}<t_{3}$ such as $t_{1}>\max _{i \leq N} h\left(\mathcal{D}_{i}\right)$ :

$$
\frac{N}{4} \min _{i \leq N} \int_{3}|\nabla 2|^{1} \leq\left[\int_{t_{2}}^{t_{3}} \frac{d t}{\mu^{2}(t) \theta(t)}\right]^{-1} \exp \left(-2 \pi N \int_{t_{1}}^{t_{2}} \frac{d t}{\theta(t)}\right)
$$

where $\mu(t)=\max _{m \in \Sigma_{h}(t)}|\nabla f| f(m)$.

Now we suppose that $x: M \rightarrow \mathbf{R}^{n}$ is a proper minimal immersion. Then $x_{k}(m), 1 \leq k \leq n$, are harmonic functions on $M$ and Lemma A.1. yields that $h(m)=|\nabla f| x(m)$ is an exhausting function. We observe that

$$
\nabla 1 h(m)=(\bar{\nabla} 1|\nabla f| x(m))^{\top}=\frac{x^{\top}(m)}{|\nabla f| x(m)},
$$

and hence,

$$
|\nabla f| \nabla 1 h(m)=\frac{|\nabla f| x^{\top}(m)}{|\nabla f| x(m)} \leq 1
$$

everywhere in $M$, and after applying Cauchy's inequality,

$$
\ln \frac{b}{a} \leq\left(\int_{a}^{b} \frac{\theta(t) d t}{t^{2}}\right)^{1 / 2}\left(\int_{a}^{b} \frac{d t}{\theta(t)}\right)^{1 / 2}
$$

we obtain

$$
\int_{a}^{b} \frac{d t}{\theta(t)} \geq\left(\ln \frac{b}{a}\right)^{2}\left[\int_{a}^{b} \frac{d t}{t^{2}} \int_{\Sigma_{t}}|\nabla f| \nabla 1 h\right]^{-1}=\left(\ln \frac{b}{a}\right)^{2}\left[\int_{B_{h}(b) \backslash B_{h}(a)} \frac{1}{|\nabla f| x^{2}}\right]^{-1}
$$


Substituting in (5.3) the coordinate function $x_{k}(m)$ instead of $f(m)$ arrive at

$$
\min _{1 \leq i \leq N} \int_{B_{h}\left(t_{1}\right) \cap \mathcal{D}_{i}}|\nabla f| \nabla 1 x_{k}^{2} \leq \frac{4 t_{3}^{2}\left(V\left(t_{3}\right)-V\left(t_{2}\right)\right)}{N \ln ^{2}\left(t_{3} / t_{2}\right)} \exp \left(-\frac{2 \pi N \ln ^{2}\left(t_{2} / t_{1}\right)}{V\left(t_{2}\right)-V\left(t_{1}\right)}\right) .
$$

Here

$$
V(t)=\int_{B_{h}(t)} \frac{1}{|x|^{2}} .
$$

This quantity have been used by the authors in the paper [24] for estimation of the extremal length of a family of curves on minimal surface. In fact, the asymptotic behaviour of $V(t)$ at infinity can describe in terms of the integral-geometrical invariants of minimal submanifolds. In recent paper [35] the following property of $V(t)$ has been established. Let $\mathcal{M}$ be a $p$-dimensional properly immersed minimal surface in $\mathbf{R}^{n}$ such that

$$
V(t) \equiv \int_{M(t)} \frac{1}{|x(m)|^{p}}=O(\ln t), \quad \text { as } t \rightarrow \infty
$$

where $M(t)=\{m: 1<|x(m)|<t\}$. Then [35] there exist the limits

$$
V_{p}(\mathcal{M}) \equiv \lim _{t \rightarrow \infty} \frac{V(t)}{\omega_{p} \ln t}=\lim _{t \rightarrow \infty} \frac{p \operatorname{Area}_{p}(M(t))}{\omega_{p} t^{p}}
$$

where $\omega_{p}$ is the $(p-1)$-dimensional measure of a unit sphere $S^{p-1}(1)$.

Remark 2. In particularly (see [35]), if $\mathcal{M}$ is a two-dimensional properly immersed surface of finite total Gaussian curvature then $V_{2}(\mathcal{M})=\ell$, where $\ell$ is the number of ends of $\mathcal{M}$. Moreover, it was shown in [35] that properly immersed $p$-dimensional minimal submanifolds with $V_{p}(\mathcal{M})<\infty$ have the finite number of topological ends. On the other hand, for the twodimensional helicoid one holds $V_{2}(\mathcal{M})=+\infty$ while its Eulerian characteristic and the number $\ell$ are finite. We don't also know whether $V_{2}(\mathcal{M})<+\infty$ yields finiteness of the total Gaussian curvature of a minimal surface $\mathcal{M}$.

In this paragraph we study relations between $V_{2}(\mathcal{M})$ and the number of humps which can be cut off from a two-dimensional minimal surface by a system of hyperplanes. In this connection, we notice that even if $\mathcal{M}$ be the two-dimensional catenoid then there are planes which don't cut off any humps from the surface. Really, it is sufficient to consider a plane which is orthogonal to the axis of the catenoid. Therefore, we need the following 
Definition 12. A direction $e \in \mathbf{R}^{n}$ is called regular for a surface $\mathcal{M}$ if $\mathcal{M}$ doesn't contain in a hyperplane othogonal to $e$ and all sections of $\mathcal{M}$ by hyperplanes $\Pi \perp e$ don't contain compact components (here and henceforth we mean by "sections of $\mathcal{M}$ etc" the words "the preimage of sections of $x(M)$ etc" if the surface $\mathcal{M}$ doesn't embedded).

For such two hyperplanes $\Pi_{1}$ and $\Pi_{2}$ we denote by $N\left(\Pi_{1} ; \Pi_{2}\right)$ the number of components of $\mathcal{M}$ lying outward of the slab with a boundary $\Pi_{1} \cup \Pi_{2}$.

We notice, that a hump must have noncompact boundary.

Theorem 5.4. Let $\mathcal{M}$ be a two-dimensional properly immersed minimal surface in $\mathbf{R}^{n}$ with finite projective 2-volume $V_{2}(\mathcal{M})$. Let e be a regular direction. Then for any hyperplanes $\Pi_{1}$ and $\Pi_{2}$ orthogonal to $e$

$$
N\left(\Pi_{1} ; \Pi_{2}\right) \leq 2 V_{2}(\mathcal{M}) .
$$

Proof. Really, let $x_{1}$ be corresponding to $e$ coordinate function $x_{1}(m)=$ $\langle u, e\rangle x(m) e$. Then $\mathcal{M}$ doesn't contain in any hyperplane which is orthogonal to $e$ and it follows that $x_{1}(m)$ does not constant. Withot loss of generality we can arrange that $\Pi_{i}$ is defined by $x_{1}=(-1)^{i} a$ for some $a>0$ and consider the subharmonic function $f(m)=\left|x_{1}(m)\right|$. We fix $t_{1}>a$ and denote by $J>0$ the left part of (5.5). Given $\varepsilon>0$, we find $t_{2}$ to be sufficient large such that

$$
V(t)<2 \pi\left(V_{2}(\mathcal{M})+\varepsilon\right) \ln t .
$$

Hence, for any $t>t_{2}$ and from (5.3),

$$
J<\frac{4 t_{3}^{2} t_{2}^{-N /\left(V_{2}(\mathcal{M})+\varepsilon\right)}}{N \ln \left(t_{3} / t_{2}\right)},
$$

where $t_{3}>t_{2}$ and $N=N\left(\Pi_{1} ; \Pi_{2}\right)$.

We choose now $t_{3}=2 t_{2}$. Then $t_{2} \rightarrow \infty$ gives

$$
2-\frac{N}{V_{2}(\mathcal{M})+\varepsilon} \geq 0,
$$

and (5.8) is proved.

5.4. In this paragraph we deal with the minimal surfaces $\mathcal{M}$ of finite topological type. This means that $\mathcal{M}$ is realized by a minimal immersion of some compact manifold $M$ of genus $g$ with a finite number $\ell$ of points removed. The last points is called the ends of $M$. 
Definition 13. Let $f(m)$ be a harmonic function on $M$. Due to [28] we define the index of the function $f(m)$ at a critical point $m_{0} \subset \mathcal{Z}(f)$ to be the number

$$
\operatorname{ind}\left(m_{0}\right)=\frac{\sigma}{2}-1
$$

where $\sigma$ is the number of spliting continua of the set $\{m \in M: f(m)=$ $f\left(m_{0}\right), m \neq m_{0}$ and $m$ is sufficiently near to $\left.m_{0}\right\}$.

It has been shown in [28] that ind $\left(m_{0}\right)$ is a positive integer provided that $f$ is not a constant.

Theorem 5.5. Let $\mathcal{M}$ be a two-dimensional properly immersed minimal surface in $\mathbf{R}^{n}$ of finite topological type, $e$ is a regular direction and $x_{1}$ be the corresponding coordinate function. If $V_{2}(\mathcal{M})<+\infty$ then the number of critical points $\left\{a_{i}\right\}$ of $x_{1}(m)$ is finite. Moreover,

$$
\sum_{j} \operatorname{ind}\left(a_{j}\right) \leq V_{2}(\mathcal{M})-\chi(M)
$$

where $\chi(M)$ is the Eulerian characteristic of $M$.

Remark 3. When $M$ is homeomorphic to a sphere with $\nu$ points removed this assertions was proved in [22].

Proof. We denote by $\mathcal{N}(t)$ the number of components of $\left\{m \in M: x_{1}(m)>\right.$ $t\}$. By virtue of the maximum principle for the coordinate functions of minimal surfaces, $\mathcal{N}(t)$ is nondecreasing integer-valued function for $t \in \mathbf{R}^{n}$, and for any critical value $a_{i}$ we have

$$
\lim _{t \rightarrow x_{1}\left(a_{i}\right)+0} \mathcal{N}(t)-\lim _{t \rightarrow x_{1}\left(a_{i}\right)-0} \mathcal{N}(t) \geq 1 .
$$

Really, the level set $\left\{m \in M: x_{1}(m)=a_{i}\right\}$ is a union of continua $\gamma$ and by virtue of assumption of the regularity of the coordinate function, none of $\gamma$ 's is compact. We write

$$
\mathcal{N}(+\infty) \equiv \lim _{t \rightarrow+\infty} \mathcal{N}(t) .
$$

We also observe that finiteness of the projective volume of $\mathcal{M}$ yields parabolic conformal type of $\mathcal{M}$ (see [24]). Thus, by the Phragmen-Lindelöf theorem applied to the harmonic function $x_{1}(m)$ we can suppose that all humps of $\left\{m \in M \mid x_{1}(m)>t\right\}$ are unbounded at the positive $x_{1}$-direction. Then finiteness of the number of critical points $a_{i}$ of $x_{1}$ follows from (5.10) 
and finiteness of the number $\mathcal{N}$ of asymptotic tracts of $\left|x_{1}(m)\right|$. The last property is a consequence of (5.8).

Our proof of (5.9) is an appropriate generalization of the corresponding estimate in [22] for zero-genus minimal surfaces.

We choose $c$ to be a positive number which is greater than the absolute value of any critical value $x_{1}\left(a_{j}\right)$. It follows from conformality of the Gauss map of a twodimensional minimal surface that it has locally finite multiplicity. This property provides (see [2, Theorem 18.5.4]) existence of the regular part $M^{\prime}$ of $M$, i.e. such a smooth compact submanifold of $M$ (with nonempty boundary) which satisfies the following conditions

1. the topological type of $M^{\prime}$ coincides with the same of $M: \chi\left(M^{\prime}\right)=$ $\chi(M)$;

2. every boundary component of $M$ is a finite family of alternating $x_{1}(m)$ level-curves $\gamma_{i}\left(x_{1}= \pm c\right)$ and the gradient-curves $\Gamma_{i}$ (i.e. the curves of the most increasing of $x_{1}(m)$ );

3. the number $\ell$ of all components of $\partial M^{\prime}$ coincides with the number of ends of $M$.

From regularity of the $e$-direction follows that each $\gamma_{i}$ is a simple open curve. As a consequence, every component of $\partial M^{\prime}$ contains of an even number of $\gamma_{i}$ and the same number of $\Gamma_{j}$.

We consider the decomposition of the following two parts of $M$ into open components

$$
\left\{x_{1}(m)>c\right\}=\cup_{i=1}^{N^{+}} \mathcal{O}_{i}^{+} \quad, \quad\left\{x_{1}(m)<-c\right\}=\cup_{j=1}^{N^{-}} \mathcal{O}_{j}^{-} .
$$

Since the set $\left\{\left|x_{1}(m)\right|>c\right\}$ doesn't contain any critical points, each $\mathcal{O}_{i}^{ \pm}$ is homotopically equivalent to a two-dimensional disk. Furthermore, one can determinate a bijection between the $\operatorname{arcs} \gamma_{i}$ and the components $\mathcal{O}_{i}^{ \pm}$. It follows from (5.8) and the alternating property of $\gamma_{i}$ and $\Gamma_{j}$ that

$$
N^{+}=N^{-}=\mathcal{N}(+\infty)=\frac{\mathcal{N}}{2} \leq V_{2}(\mathcal{M}) .
$$

Let $M_{0}=M^{\prime} \# M^{\prime}$ be the result of pasting together of two copies of $M^{\prime}$ along $\partial M^{\prime}$ and consequent contracting of each curve $\gamma_{i}$ and its copy into a point $G_{i}$. Then (see [34], Section 5, ex. 5), the genus $g_{0}$ of $M_{0}$ can be expressed by

$$
g_{0}=2 g+\ell-1,
$$


where $\ell$ is a number of the components of $\partial M^{\prime}$.

By virtue of the definition of $M_{0}$ the coordinate function $x_{1}(m)$ can be canonically lifted up to the well-defined on $M_{0}$ function $f$. Then $f(\xi)$ is smooth everywhere on $M_{0} \backslash G$, where $G=\left\{G_{i}\right\}$. Moreover, all of $G_{j}$ 's are the points of strong maximum and minimum of $f(\xi)$.

For the Eulerian characteristic we have from (5.11)

$$
\chi\left(M_{0}\right)=2-2 g_{0}=2(2-2 g-\ell)=2 \chi\left(M^{\prime}\right)=2 \chi(M) .
$$

We can lift by a natural way the gradients field $\nabla x_{1}(m)$ up to the continuous vector field $X(m) \equiv \nabla f$ on $M_{0} \backslash G$. Then the set of singular points of $X(m)$ consists of: a) the critical points $\left\{a_{i}\right\}_{i=1}^{k}$ and its doubles $\left\{a_{i}^{\star}\right\}_{i=1}^{k}$; and b) the singular points $G_{j}$.

Let $\operatorname{ind}_{\xi} X$ denotes the rotational number (index) of the field $X$ at $\xi[27$, $\S 5]$. Then at the extreme points we have

$$
\operatorname{ind}_{G_{j}} X=1 \text {. }
$$

On the other hand, by virtue of the definition of the index of harmonic function (see also $[27, \S 6]$ ) it is easily seen

$$
\operatorname{ind}_{a_{i}} X=\operatorname{ind}_{a_{i}^{\star}} X \equiv \operatorname{ind}_{a_{i}}\left(\nabla x_{1}\right)=-\operatorname{ind}\left(a_{i}\right) .
$$

Indeed, the last identity follows from analysis of a sufficient small neighbourhood of the critical point $a_{i}$ of a harmonic function.

Thus, we can apply the Poincare-Hopf theorem and from (5.13), (5.14) we obtain

$$
\begin{gathered}
\chi\left(M_{0}\right)=\sum \operatorname{ind}_{a_{i}} X+\sum \operatorname{ind}_{a_{i}^{\star}} X+\sum_{j=1}^{\mathcal{N}} \operatorname{ind}_{G_{j}} X= \\
-2 \sum \operatorname{ind}\left(a_{i}\right)+\mathcal{N} .
\end{gathered}
$$

Hence, by (5.12) we arrive at

$$
\sum \operatorname{ind}\left(a_{i}\right)=\frac{\mathcal{N}}{2}-\chi(M) \leq V_{2}(\mathcal{M})-\chi(M),
$$

and the theorem is proved.

5.5. To present some applications of these theorems we first suppose that $\mathcal{M}$ is a properly immersed plane in $\mathbf{R}^{n} 3$, i.e. $\chi(M)=\chi\left(\mathbf{R}^{n} 2\right)=1$. 
Definition 14. Let $V$ be a 2-dimensional plane in $\mathbf{R}^{n} 3$ and $\pi_{V}: \mathbf{R}^{n} 3 \rightarrow$ $V$ be the orthogonal projection. A surface $\mathcal{M}=(M ; u)$ is said to be proper with respect to $V$ if for any sequence $\left\{m_{k}\right\} \subset M$ without accumulation points in $M$ the sequence $\pi_{V} \circ u\left(m_{k}\right)$ hasn't any accumulation point in $V$. Other words, the composition $\pi_{V} \circ u$ is proper mapping.

Given a plane $V$ in $\mathbf{R}^{n} 3$ denote by $n(v)$ the algebraic multiplicity of the orthogonal projection of $\mathcal{M}$ onto $V$ at $v$.

Theorem 5.6. Let $\mathcal{M}$ be a two-dimensional minimal properly immersed plane in $\mathbf{R}^{n} 3$ and $\mathcal{M}$ be proper with respect to a two-dimensional plane $V$. Then either $\mathcal{M}$ is a plane, or

$$
\liminf _{R \rightarrow \infty} \frac{1}{\ln R} \int_{1}^{R} \frac{d t}{t} \int_{0}^{2 \pi} n\left(t e^{i \theta}\right) d \theta \geq 8 .
$$

Proof. In [24] (see also [35]) was proved that

$$
V_{2}(\mathcal{M}) \leq \liminf _{R \rightarrow \infty} \frac{1}{4 \ln R} \int_{1}^{R} \frac{d t}{t} \int_{0}^{2 \pi} n\left(t e^{i \theta}\right) d \theta .
$$

Hence $\mathcal{M}$ has finite projective volume $V_{2}(\mathcal{M})$. Let $\sigma: M \rightarrow \mathrm{S}^{2}$ be the Gauss map and $\mathcal{M}$ be different from a plane. Then $\sigma(M)$ has nonempty interior int $\sigma(M)$. We choose arbitrary $\nu \in \operatorname{int} \sigma(M)$. Thus the corresponding coordinate function

$$
f(m)=\langle u, e\rangle x(m) \nu=\nu_{1} x_{1}(m)+\nu_{2} x_{2}(m)+\nu_{3} x_{3}(m)
$$

has at least one critical point $m_{0}$ corresponding to $\sigma\left(m_{0}\right)=\nu$. We notice, that by virtue of our assumption $\chi(\mathcal{M})=1, \nu$ is a regular direction provided $\mathcal{M}$ doesn't consist in a hyperplane. Therefore, we have

$$
\operatorname{ind}\left(m_{0}\right) \geq 1 \text {. }
$$

Now our statement follows from $\chi(\mathcal{M})=1,(5.17),(5.16)$ and (5.9).

As a consequence of this theorem we obtain a new proof of the Bernstein's theorem.

Corollary 5.7. The only entire graphs of minimal surfaces are the planes.

Proof. We observe that $n(v) \equiv 1$ for a graph and therefore

$$
\liminf _{R \rightarrow \infty} \frac{1}{\ln R} \int_{1}^{R} \frac{d t}{t} \int_{0}^{2 \pi} n\left(t e^{i \theta}\right) d \theta=2 \pi<8
$$


Another consequence gives a quantative form of the previous result.

Corollary 5.8. Let $\mathcal{M}$ be an immersed minimal surface of finite topological type which is homeomorphic to a compact Riemannian surface of genus $g$ with $l$ points removed. Then in the given above notations:

$$
\liminf _{R \rightarrow \infty} \frac{1}{\ln R} \int_{1}^{R} \frac{d t}{t} \int_{0}^{2 \pi} n\left(t e^{i \theta}\right) d \theta \geq 2(l+3-g) .
$$

\section{References.}

[1] AhlFors, L.: Lectures on quasiconformal mappings. Toronto-New YorkLondon: Van Nostrand Math. Studies, 1966.

[2] BaKelman, I.YA.; Verner, A.L.; Cantor, B.E.: Introduction in the geometry "in general". Moscow: Nauka, 1973.

[3] BeRnstein, S.N.: Sur un theoreme de geometrie et ses applications aux equations aux derivees partielles du type elliptique, Comm. Soc. Math. Kharkov (2) 15(1915-1917) 38-45.

[4] Cheng, S.Y.; YAU,S.T.: Differential equations on Riemannian manifolds and their geometric applications, Comm. Pure Appl. Math. 28(1975), 201-228.

[5] Cheng, S.Y.; YAU, S.T.: Maximal space-like hypersurfaces in the LorentzMinkowski spaces, Ann. of Math., V. 104(1976), 407-419.

[6] Courant, R.: Dirichlet's principle, conformal mappings and minimal surfaces. Interscience, 1950.

[7] Federer, H.: Geometric measure theory. Berlin-Heidelberg-New York: Springer 1969.

[8] Gilbarg, D.: Trudinger, N.S.: Elliptic partial differential equations of second order. 2nd Ed., Berlin-Heidelberg-New York-Tokyo: Springer, 1983.

[9] GRIGOR'YAN, A.A.: On Liouville theorems for harmonic functions with finite Dirichlet integral, Math. USSR Sbornik, 60(1988) 485-504.

[10] Heinonen, J.; KilpeläInen, T.; Martio, O.: Nonlinear potential theory of degenerate elliptic equations. Oxford Univ. Press, London, 1993.

[11] HildeBRANDT, S.: Maximum principles for minimal surfaces and for surfaces of continuous mean curvature, Math. Z. 128(1972) 253-269.

[12] HildebrandT, S.: Liouville theorems for harmonic mappings and approach to Bernstein theorems, Ann. Math. Stud., 102(1982) 107-131. 
[13] Hayman, W.K.; Kennedy, P.B.: Subharmonic functions. Acad. Press, London-New York, 1976.

[14] Hoffman, D.; MeEKs, W.H.: Embedded minimal surfaces of finite topology, Ann.of Math. 131(1990) 1-34.

[15] Hoffman, D.; MeEks, W.H.: Minimal surfaces based on the catenoid, Amer. Math. Month. 97(1990) 702-730.

[16] Klyachin, V.A.: Estimate of spread for minimal surfaces of arbitrary codimension, Sibirsk. Mat. Zh., 33(1992), N5, 201-207.

[17] Kobayashi, Sh.; Nomizu, K.: Foundations of differential geometry. Vol.2, Interscience, 1969.

[18] LINDQVIST, P.: On the definition and properties of p-superharmonic functions. J. Reine Angew. Math., 365(1986), 67-75.

[19] MacLane, G.R.: Asymptotic values of holomorphic functions. Huston 1, Texas: Rice Univ. Stud. Monograph in Math., 1963.

[20] Miklyukov, V.M.: On a new approach to Bernstein's theorem and related questions for equations of minimal surfaces type, Math. sbornik., N2, 108(1979), 268-289.

[21] Miklyukov, V.M.: On the asymptotic properties of subsolutions of quasilinear equations of elliptic type and mappings with bounded distortion, Math. sbornik, N1 111(1980), 42-66.

[22] Miklyukov, V.M.: Some singularities in the behavior of equations of minimal-surface type in unbounded domains, Math. sbornik, N1 116(1981) 72-86.

[23] Miklyukov, V.M.: Asymptotic tracts of subharmonic function on a manifold and exterior structure of minimal surfaces, Abstr. of Conf. "Geometry and Analysis", Novosibirsk, 14-16 Nov. 1989, Novosibirsk, 1989, 54.

[24] Miklyukov, V.M.; TKacheV, V.G.: On structure in large of externally complete minimal surfaces in $R^{n}$, Izvest. Vuzov. Mathematika, 7 (1987) 30-36.

[25] Miklyukov, V.M.; TKAChEV, V.G.: Some properties of tubular minimal surfaces of arbitrary codimension, Math. sbornik, N9 180(1989) 1278-1295.

[26] Miklyukov, V.M.; Vedenyapin, A.D.: Extrinic dimension of tubular minimal hypersurfaces, Math. sbornik, N2 131(1986) 240-250.

[27] MilnoR, J.W.: Topology from the differentiable viewpoint. The Univ. Press of Virginia, 1965.

[28] Morse, M.: Topological methods in the theory of functions of a complex variable. Princeton, 1947.

[29] Nitsche, J.C.C.: Lectures on minimal surfaces. Vol 1., Cambridge Univ. Press, Cambridge-New York-New Rochelle-Melbourne-Sydney, 1989. 
[30] Osserman, R.: A survey of minimal surfaces. New York: Dover Publications, 1987.

[31] Reshetnyak, Yu.G.: The mappings with bounded distortion in space. Novosibirsk: Nauka, 1982.

[32] Simon, L.: A Holder estimate for quasiconformal mappings between surfaces in Euclidean space, with application to graphs, having quasiconformal Gauss map, Acta math. 139(1977) 19-51.

[33] Simons, J.: Minimal varieties in Riemannian manifolds, Ann. of Math. 88(1968) 62-105.

[34] Springer, J.: Introduction to Riemann surfaces. Addisson-Wesley Publ. Comp., 1957.

[35] TKachev, V.G.: Finiteness of the number of ends of minimal submanifolds in Euclidean space, Manuscr. Math., (82)1994, 313-330.

[36] TkacheV, V.G.: External geometry of p-minimal surfaces, Abstr. Pacific Rim Conf., Singapore, 12-17 Decem. 1994, 17-18.

[37] YAU S.T.: Harmonic functions on complete Riemannian manifolds, Comm.Pure Appl.Math., 28(1975), 201-228.

Received May 6, 1995.

Department of Mathematical Analysis

VOLGOGRAD UNIVERSITY

2-Ya PRODOLNAYA 30

VOLGOGRAD 400062

RUSSIA

E-MAIL: MIKLUKOV@MATH.VGU.TSARITSYN.SU

E-MAIL:TKACHEV@MATH.VGU.TSARITSYN.SU 